\title{
Regulation of Neutrophil Degranulation and Cytokine Secretion: A Novel Model Approach Based on Linear Fitting
}

\author{
Isabelle Naegelen, Nicolas Beaume, Sébastien Plançon, Véronique Schenten, \\ Eric J. Tschirhart, and Sabrina Bréchard
}

Calcium Signalling and Inflammation, Life Sciences Research Unit, University of Luxembourg, 162A Avenue de la Fä̈encerie, 1511 Luxembourg, Luxembourg

Correspondence should be addressed to Sabrina Bréchard; sabrina.brechard@uni.lu

Received 11 August 2015; Accepted 29 September 2015

Academic Editor: Eileen Uribe-Querol

Copyright (c) 2015 Isabelle Naegelen et al. This is an open access article distributed under the Creative Commons Attribution License, which permits unrestricted use, distribution, and reproduction in any medium, provided the original work is properly cited.

\begin{abstract}
Neutrophils participate in the maintenance of host integrity by releasing various cytotoxic proteins during degranulation. Due to recent advances, a major role has been attributed to neutrophil-derived cytokine secretion in the initiation, exacerbation, and resolution of inflammatory responses. Because the release of neutrophil-derived products orchestrates the action of other immune cells at the infection site and, thus, can contribute to the development of chronic inflammatory diseases, we aimed to investigate in more detail the spatiotemporal regulation of neutrophil-mediated release mechanisms of proinflammatory mediators. Purified human neutrophils were stimulated for different time points with lipopolysaccharide. Cells and supernatants were analyzed by flow cytometry techniques and used to establish secretion profiles of granules and cytokines. To analyze the link between cytokine release and degranulation time series, we propose an original strategy based on linear fitting, which may be used as a guideline, to (i) define the relationship of granule proteins and cytokines secreted to the inflammatory site and (ii) investigate the spatial regulation of neutrophil cytokine release. The model approach presented here aims to predict the correlation between neutrophilderived cytokine secretion and degranulation and may easily be extrapolated to investigate the relationship between other types of time series of functional processes.
\end{abstract}

\section{Introduction}

Historically, neutrophils were described as simple professional killers of invading pathogens to the human organism [1]. In this regard, it was considered that only the release of various antimicrobial and cytotoxic proteins synthesized and distributed into different types of granules participated to the innate immune response mediated by neutrophils. Granule types have been characterized to be readily mobilized upon an inflammatory stimulus at the plasma membrane in reverse order to their formation according to the formedfirst-released-last model [2]. Indeed, in the different stages of neutrophil development, azurophil granules are formed first followed by specific granules, gelatinase granules, and, lastly, secretory vesicles, which are the most easily mobilized organelles in the mature neutrophils.
Due to recent progress, this classical view has been expanded by the acknowledgment that appropriately activated neutrophils constitute a substantial source of a variety of secreted cytokines supporting a direct contribution of these cells in the regulation framework of the adaptive immune response [3-5]. Neutrophils not only are a source of de novo synthesized cytokines dependent on gene induction but also have the capacity to express cytokines at a basal level from preformed stores [2]. However, precise intracellular localization of these packaged cytokines and mechanisms underlining their secretion remain largely elusive. The widely accepted assumption is that multiple secretory pathways coexist in neutrophils allowing the regulated release of diverse proinflammatory mediators [6]. Preformed cytokines are instantly released upon ligand-receptor signaling during the so-called "regulated exocytosis" process [7] whereas de 
novo synthesized cytokines may be released after trafficking via recycling endosomes during the mechanism referred to as "constitutive exocytosis" $[8,9]$. Additionally, variations of these two main classical secretion pathways have also been reported [10]. These distinct processes selectively control the combination of granule proteins and cytokines released into the local microenvironment from neutrophils over a temporal and spatial range and are thus regulatory mechanisms important for the onset and resolution of inflammation enabling the development of an appropriate inflammatory response [11].

It is now largely recognized that neutrophil-derived granule proteins and cytokines contribute to the maintenance of the inflammatory response and, when excessively secreted, to the ongoing process of tissue damage leading to the development of many chronic inflammatory disorders such as inflammatory bowel diseases [12], rheumatoid arthritis [13], chronic obstructive pulmonary disease [14], and atherosclerosis [15]. Determination of the regulatory mechanisms mediating the different patterns of cytokine trafficking and release may create opportunities to define new targets or strategies to selectively reduce cytokine secretion in clinical diseases.

Therefore, we selected relevant cytokines secreted by neutrophils, described to contribute to the development of chronic inflammatory diseases, in order to investigate their release in combination to degranulation upon stimulation with bacterial lipopolysaccharide (LPS).

Here, we propose an appealing model based on a linear fitting approach of cytokine secretion and degranulation giving a first basis for deeper understanding of the relationship between these two processes. It also provides a predictive view on the distribution of cytokines in neutrophils and offers an outstanding starting point to target future research on release mechanisms involved in inflammatory processes.

\section{Materials and Methods}

2.1. Purification of Human Neutrophils. Peripheral blood of healthy volunteers was collected in EDTA-containing tubes (BD Vacutainer, BD Biosciences, Erembodegem, Belgium). Samples were collected in accordance with the good clinical and ethical practices, which have been approved by the Ethics Review Panel (ERP) of the University of Luxembourg according to the "Comité National d'Ethique de Recherche" (CNER) from Luxembourg.

Neutrophils were isolated from blood samples by Polymorphprep separation procedure (Axis-Shield, Dundee, Scotland) according to manufacturer's instructions. Remaining erythrocytes in the neutrophil cell suspension were lysed for $10 \mathrm{~min}$ with red blood cell lysis buffer $\left(155 \mathrm{mM} \mathrm{NH}_{4} \mathrm{Cl}\right.$, $10 \mathrm{mM} \mathrm{KHCO}_{3}, 0.1 \mathrm{mM}$ EDTA, and $\mathrm{pH} 7.4$ [16]).

Neutrophils were washed and resuspended in PBS 1x ( $\mathrm{pH}$ 7.4). Purity of isolated neutrophils was analyzed by the BD FACSCanto II flow cytometer (BD Biosciences) using two mixtures of selection markers CD66b-FITC/CD11bPE/CD14-APC and CD15-FITC/CD16-PE/CD45-APC (Immunotools, Friesoythe, Germany) on 10,000 events in the gated population of homogenous (FSC-A versus SSC-A), single (SSC-A versus SSC-H), and living cells (negative cells for Sytox Blue staining (Invitrogen, Gent, Belgium)). Purified neutrophils are positive for all the selection markers used by flow cytometry. Human neutrophils were cultured in X-VIVO 15 medium with L-glutamine and gentamicin (Lonza) at $37^{\circ} \mathrm{C}$ and $5 \% \mathrm{CO}_{2}$ up to $24 \mathrm{~h}$ after purification.

2.2. Cell Stimulation. Purified neutrophils were stimulated with bacterial LPS from E. coli serotype O111:B4 (Sigma, Bornem, Belgium) for simulating proinflammatory conditions. For kinetic studies of cytokine secretion and degranulation, neutrophils were stimulated with $100 \mathrm{ng} / \mathrm{mL}$ LPS for $2,4,6,12$, and $24 \mathrm{~h}$ under serum-free conditions to avoid any serum component contamination, which could interfere with specific LPS-induced cell responses.

2.3. Cell Analysis by Flow Cytometry. In accordance with the literature, the most relevant markers have been selected for degranulation analysis [17]. Degranulation was determined by measuring the expression of $\mathrm{CD}$ markers characteristic for azurophil granules (CD63-PE), specific granules (CD15FITC, CD66b-FITC), gelatinase granules (CD11b-PE), and secretory vesicles (CD13-APC, CD14-APC, CD18-FITC, and CD45-APC) at the plasma membrane by flow cytometry (all antibodies are from BD Biosciences except CD14-APC from Immunotools).

IgG1-FITC, IgG2a-PE (BD Biosciences), and IgG1-APC antibodies (Immunotools) were used as negative isotype controls to place the cells in the first decade of any plot, whereas CD45-FITC, CD45-PE, or CD45-APC (BD Biosciences) single dye staining was used to set compensations. Data analysis was performed by measuring the mean fluorescence intensity (MFI) for each CD marker with BD FACSDiva software (BD Biosciences) on the gated population of granulocytes (FSCA versus SSC-A), single (SSC-A versus SSC-H), and living cells (negative cells for Sytox Blue staining (Invitrogen)). In total, 10,000 events were recorded per staining. The relative translocation of CD markers to the plasma membrane for each granule was determined by calculating the ratio between MFI of LPS-stimulated cells and nonstimulated control from the same time point.

2.4. Measurement of Cytokine Secretion by Cytometric Bead Array (CBA). Density of human neutrophils was adjusted to $10 \times 10^{6}$ cells per condition for subsequent quantitative measurement of cytokine secretion by LPS-stimulated cells, respectively. Fresh supernatants were collected and used directly for cytometric bead array (CBA, BD Biosciences) analysis. The multiplex standard curve composed of mixed cytokine standards was set up by serial dilutions according to the manufacturer's instructions. Selected capture beads were prepared and added to supernatants. The following beads were used: CCL2 (MCP1, bead D8), CCL3 (MIP1 $\alpha$, bead B9), CCL4 (MIP1 $\beta$, bead E4), CCL5 (RANTES, bead D4), ILla (bead D6), IL1b (bead B4), IL6 (bead A7), IL8 (CXCL8, bead A9), IL12b (bead E5), and TNFa (bead C4). After $1 \mathrm{~h}$ of incubation, detection reagent was added to each sample. 
After $2 \mathrm{~h}$ of incubation, samples were rinsed with wash buffer and centrifuged. Samples were washed again prior to flow cytometry analysis (BD FACSCanto II, BD Biosciences). Results were quantified using the standard curves and the Flow Cytometric Analysis Program (FCAP) Array software (Soft Flow, Minneapolis, USA).

2.5. Linear Fitting Approach via $R$ Statistical Software. Kinetic profiles of cytokine secretion and degranulation were imported into R statistical software (https://www.r-project .org/) and a linear regression approach was applied. This approach was used to find the optimal proportionality factor, namely, the slope of the model, and provide methods to evaluate the significance of our models. All ratio values between LPS-stimulated and nonstimulated control conditions (stimulation points $0,2,4,6,12$, and $24 \mathrm{~h}$ ) from the time series of cytokine secretion and degranulation were $\log _{10}$ normalized to minimize scale effect. For each granulespecific CD marker, a linear model has been fitted with the secreted cytokines. For each model, ANOVA analysis has been performed and the adjusted $R$-squared (RSQ) value and the slope of the model were retained. Models with a significant difference to the null model ( $p$ value $\leq 0.05$ ) and with a high adjusted RSQ value between degranulation marker and secreted cytokine (RSQ $\geq r$, with $r$ being determined by simulations; see Section 2) were considered as underlying a strong similarity of pattern between a secreted cytokine and a mobilized degranulation marker. Then, cytokines linked to the same degranulation marker were clustered. To visualize these clusters, time series of the degranulation marker and its relative cytokines of the cluster were plotted. To permit comparison between time series from different scales, values from the secreted cytokines were divided by the slope of their linear model.

2.6. Simulations to Determine the Optimal RSQ Threshold. To define the optimal RSQ threshold, we simulated the fitting between pairs of granule marker and cytokine with controlled perturbations between them and choose the threshold by determining at which level of perturbation the RSQ was drastically dropping. The simulations were designed as follows: time series of each granule-specific marker and cytokines were taken individually (19 time series in total) and used to simulate matching time series with more or less perturbations. For the six points of each time series $(0,2$, $4,6,12$, and $24 \mathrm{~h}$ ), a random number between $V_{i}-e$ and $V_{i}+e$ was drawn. $V_{i}$ is the $i$ th element of the time series and $e$ is a predefined constant. $e$ controls the intensity of the perturbation: the highest $e$ is the more different both profiles are expected to be. All values from 0 to 1 with a step of 0.1 were tested for $e(0,0.1,0.2$, etc., to 1$)$. Then, the RSQ of the original profile versus simulated profile was computed as mentioned in linear fitting approach via $R$ statistical software. This process was repeated 1000 times and, for each of the 19 original profiles, the average of the RSQs was computed and plotted against the $e$ value. To define the optimal RSQ threshold that defines a cut-off between linear fitting models with "high RSQ" and "low RSQ," we clustered the distribution of averaged RSQ into two groups, using a $k$-means approach (a silhouette analysis of all clustering solution with 2 to 10 clusters confirmed that using 2 clusters was the best solution, data not shown). The last element of the cluster with the highest center, namely, 0.796, was taken as RSQ threshold for our analysis.

2.7. Statistical Analysis. Statistical analyses were performed using the PRISM6 software (Graph Pad Software, La Jolla, CA, USA). When normality and homogeneity of variances were ascertained, as determined by the $F$-test, Student's $t$-test analyses were performed to establish two group comparisons. Otherwise, Mann-Whitney tests were used for two group comparisons. $p$ values $\leq 0.05$ were considered statistically significant.

\section{Results}

3.1. Cytokine Levels Secreted by Human Neutrophils upon LPS Stimulation. Since recent reports have implicated neutrophils in the development of chronic inflammatory disorders, we wanted to characterize the regulatory mechanisms in the release of neutrophil-derived products. In a first step, cytokine candidates found secreted by highly purified $(\geq 98 \%)$ neutrophils upon LPS stimulation [17] were selected for integration into our mathematical model. These cytokines have a particular relevance since they have been reported to contribute to the development of different chronic inflammatory diseases through the recruitment of diverse immune cells to the inflammatory site (Table 1).

To develop a reliable model that investigates the relationship between degranulation and cytokine secretion, different experimental data sets were generated. Time series of cytokine secretion were determined to serve as input to our model. Neutrophils were treated for $0 \mathrm{~h}, 2 \mathrm{~h}, 4 \mathrm{~h}, 6 \mathrm{~h}, 12 \mathrm{~h}$, and $24 \mathrm{~h}$ with LPS $100 \mathrm{ng} / \mathrm{mL}$ since maximal peak of secretion for cytokines was reached at this concentration (data not shown). Subsequent quantitative measurement of cytokine secretion was performed by the CBA technique. A basal secretion level of all cytokines was detected in supernatants from neutrophils under nonstimulated conditions. Secretion of most of the cytokines released into the extracellular medium was augmented with increasing time of LPS stimulation, except for IL12 $\beta$ and CCL5 whose release was not significantly affected by LPS treatment (Figure 1).

The secretion pattern was different for each cytokine in the way that different profiles could be identified. Except for ILl $\alpha$, secretion levels of cytokines were maximal $6 \mathrm{~h}$ or $12 \mathrm{~h}$ after treatment with LPS and decreased after $24 \mathrm{~h}$. Maximal cytokine secretion was observed at (i) $6 \mathrm{~h}$ LPS for CCL 3 and (ii) $12 \mathrm{~h}$ LPS for TNF $\alpha$, IL6, IL8, CCL2, IL1 $\beta$, and CCL4. Moreover, quantities of released cytokines were highly variable. TNF $\alpha$, IL1 $\alpha, \operatorname{IL} 1 \beta$, and CCL3 were only discretely secreted $(\leq 150 \mathrm{pg} / \mathrm{mL})$ whereas IL6, CCL2, and CCL4 were secreted at an intermediate level $(\sim 250-600 \mathrm{pg} / \mathrm{mL})$ and IL8 was highly secreted $(\geq 30000 \mathrm{pg} / \mathrm{mL})$.

3.2. Effect of LPS on Degranulation in Neutrophils. To collect data for the implementation of the model, the second series 
TNF $\alpha$

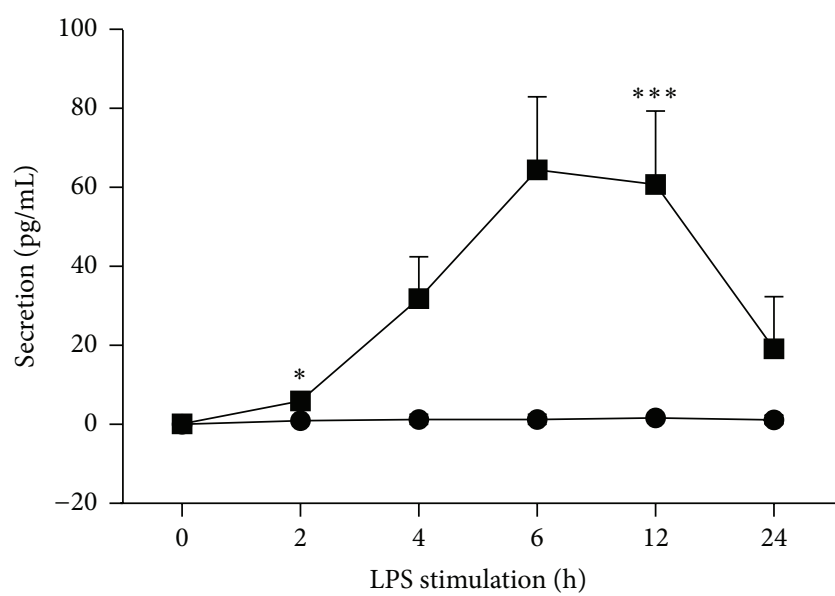

- $100 \mathrm{ng} / \mathrm{mL}$ LPS

- Control

IL1 $\beta$

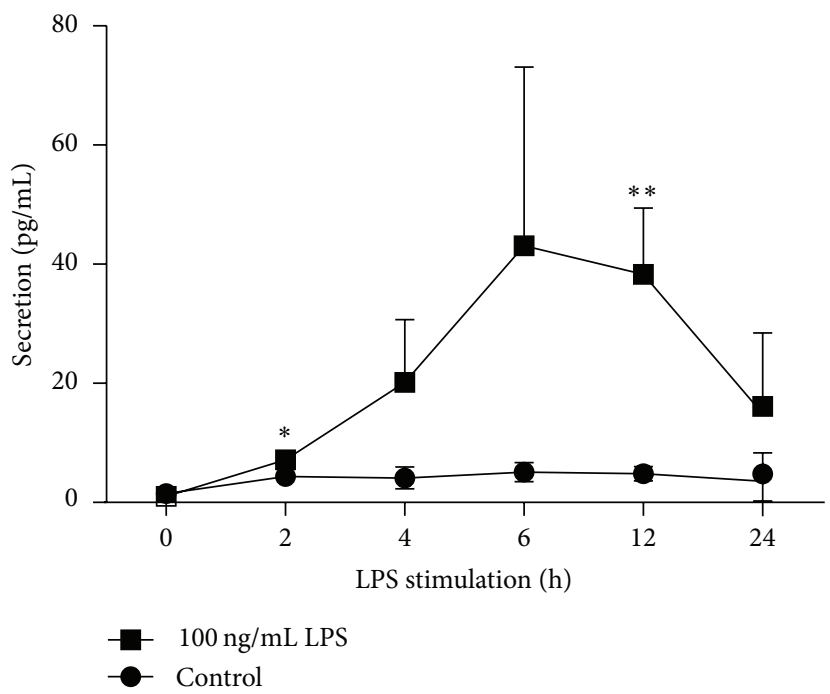

IL12 $\beta$

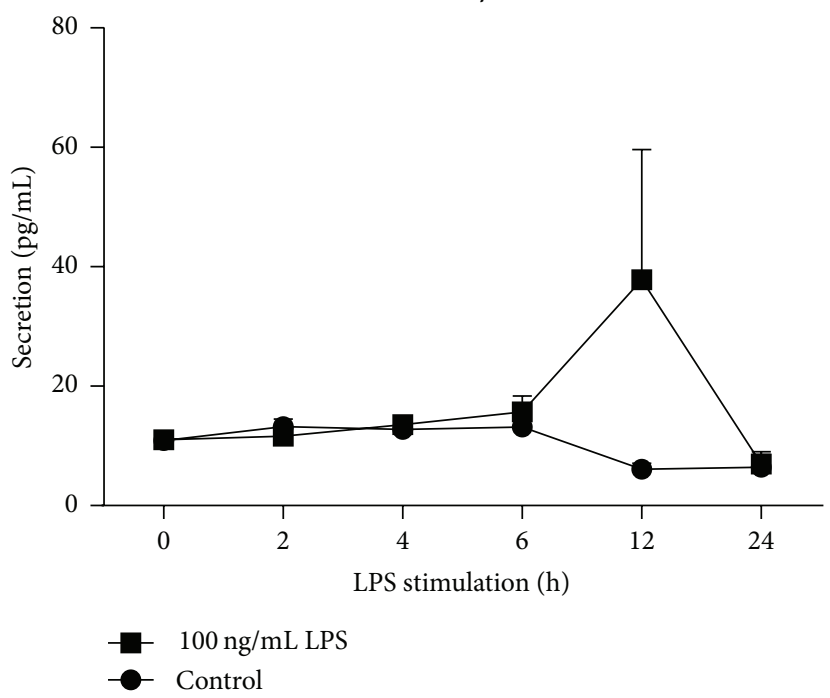

IL1 $\alpha$

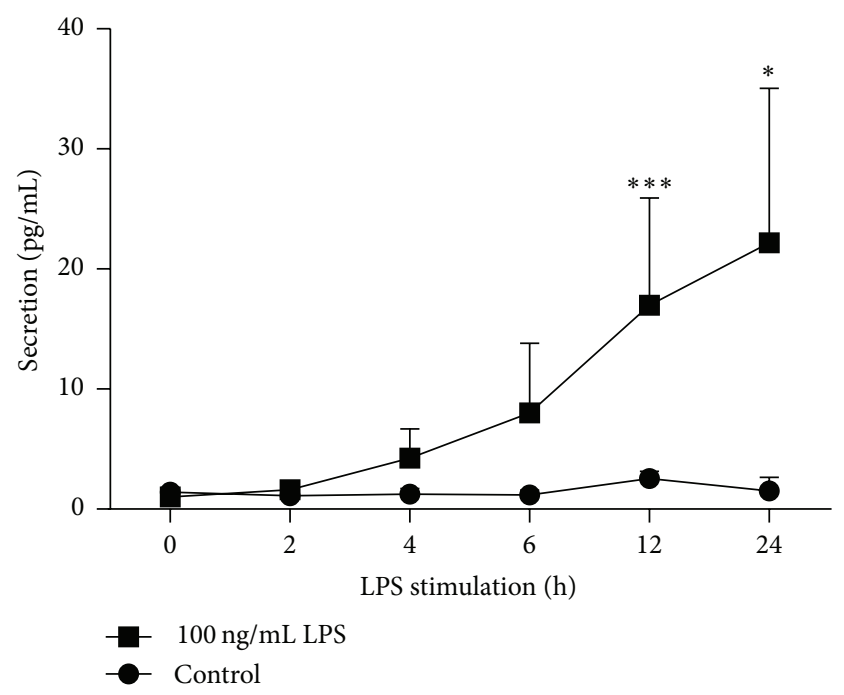

IL6

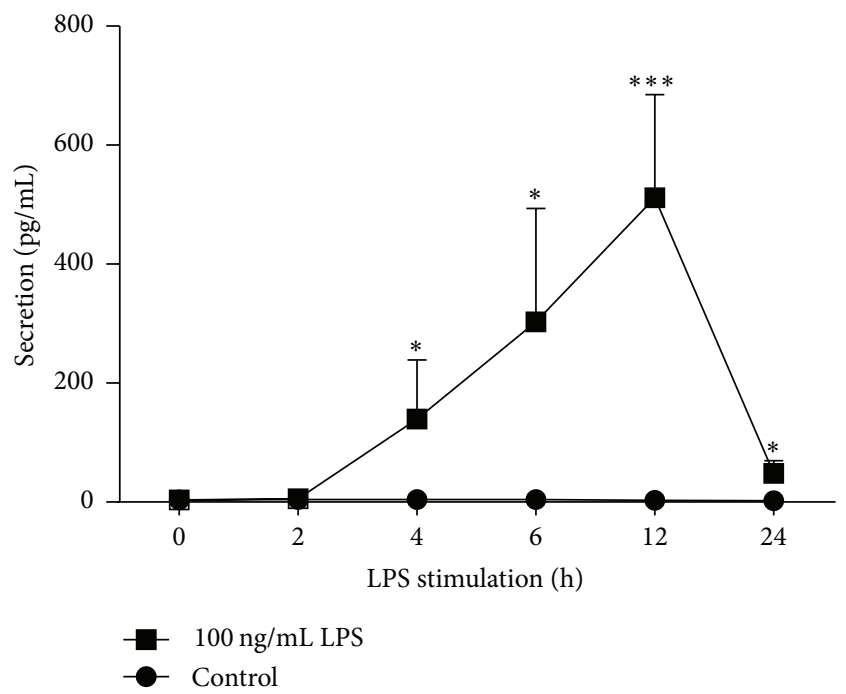

CCL2

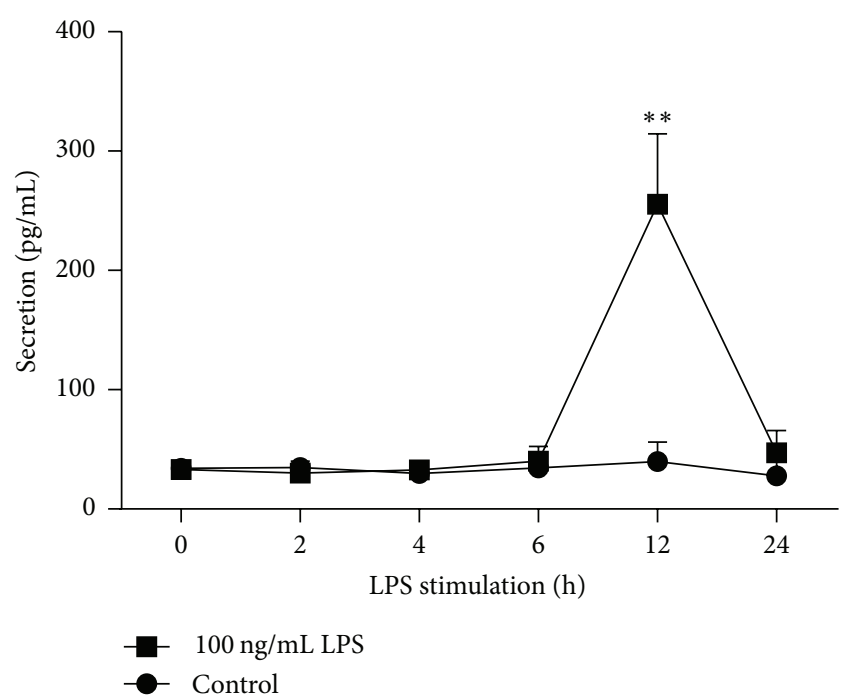

FIgure 1: Continued. 
CCL3

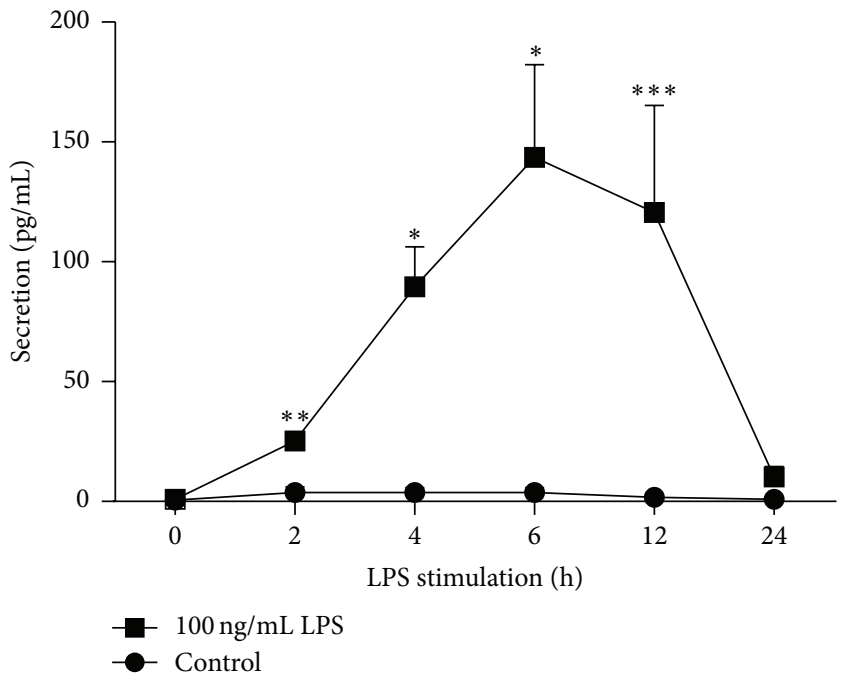

CCL5

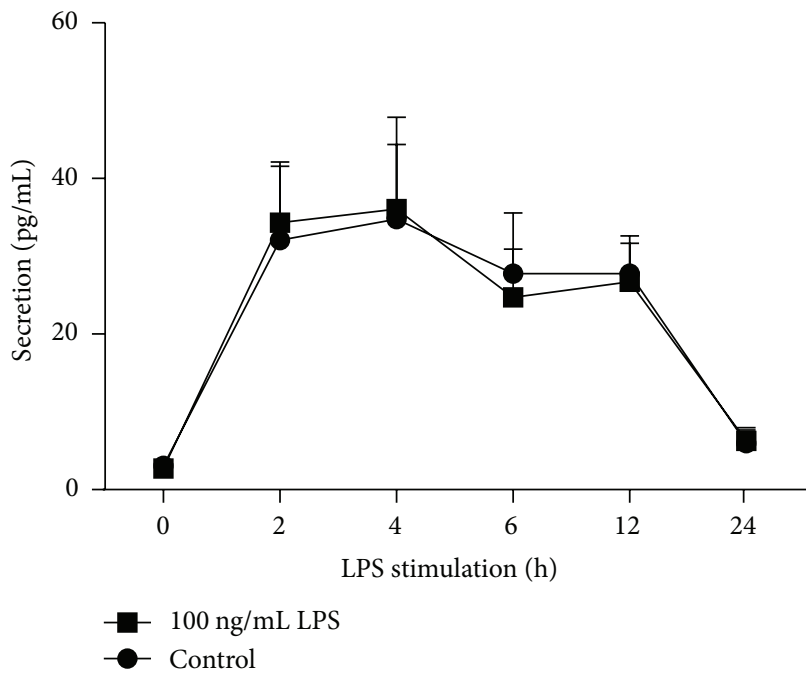

CCL4

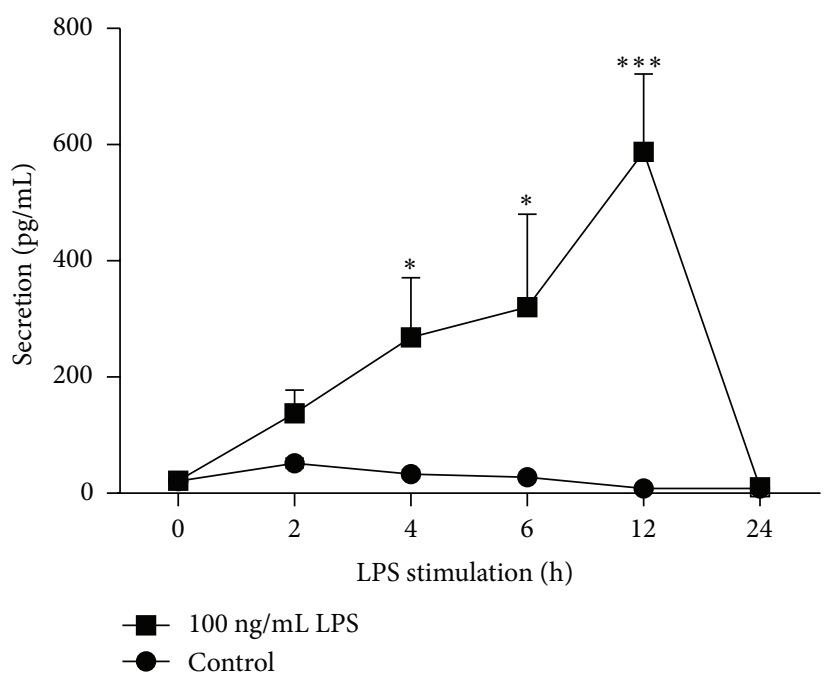

IL8

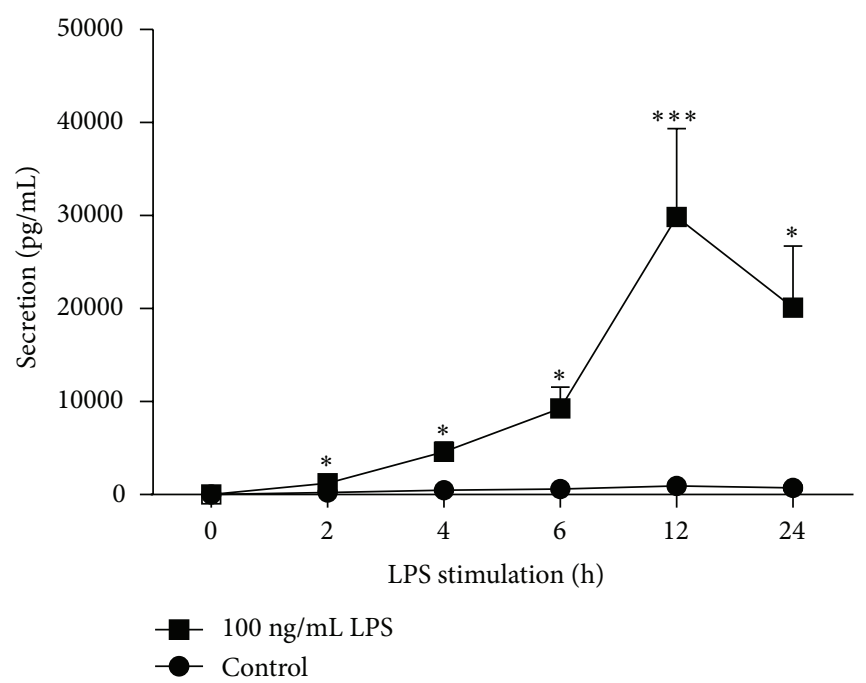

FIGURE 1: Time-dependent effect of LPS on cytokine secretion in human neutrophils. Cytokine secretion was measured by CBA upon stimulation with $100 \mathrm{ng} / \mathrm{mL}$ LPS for $0 \mathrm{~h}, 2 \mathrm{~h}, 4 \mathrm{~h}, 6 \mathrm{~h}, 12 \mathrm{~h}$, and $24 \mathrm{~h}$. Results are mean secretion $(\mathrm{pg} / \mathrm{mL}) \pm \mathrm{SEM}$ of at least 3 independent experiments, significantly different from cytokine secretion in nonstimulated control at the corresponding time: ${ }^{*} p<0.05$, ${ }^{* *} p<0.01$, ${ }^{* * *} p<0.001$, and ${ }^{* * * *} p<0.0001$.

TABLE 1: List of selected proinflammatory cytokines contributing to the development of chronic inflammatory disorders.

\begin{tabular}{lcc}
\hline Cytokine & Cell recruitment & Chronic inflammatory disorders \\
\hline TNF $\alpha$ & Monocytes, neutrophils, and dendritic cells & RA [13], IBD [18], A [19], and COPD [20] \\
IL1 $\alpha$ & Neutrophils, T cells & RA [21], IBD [22] \\
IL1 $\beta$ & Neutrophils, thrombocytes, and T cells & RA [13] \\
IL6 & Neutrophils, B cells & RA [13], A [23], and COPD [24] \\
IL12 $\beta$ & T cells & RA [25], IBD [26], and A [27] \\
CCL2 & Monocytes, dendritic cells, and memory T cells & RA [21], A [28] \\
CCL3 & Neutrophils, eosinophils, and basophils & RA [29], A [30] \\
CCL4 & Monocytes, dendritic cells, NK cells, and T cells & RA [31], IBD [32], and A [30] \\
CCL5 & Eosinophils, basophils, and T cells & RA [33], A [30] \\
IL8 & Neutrophils, macrophages, and mast cells & RA [13], A [28], and IBD [34]
\end{tabular}

$\mathrm{RA}=$ rheumatoid arthritis, $\mathrm{IBD}=$ inflammatory bowel disease, $\mathrm{A}=$ atherosclerosis, and $\mathrm{COPD}=$ chronic obstructive pulmonary disease. 

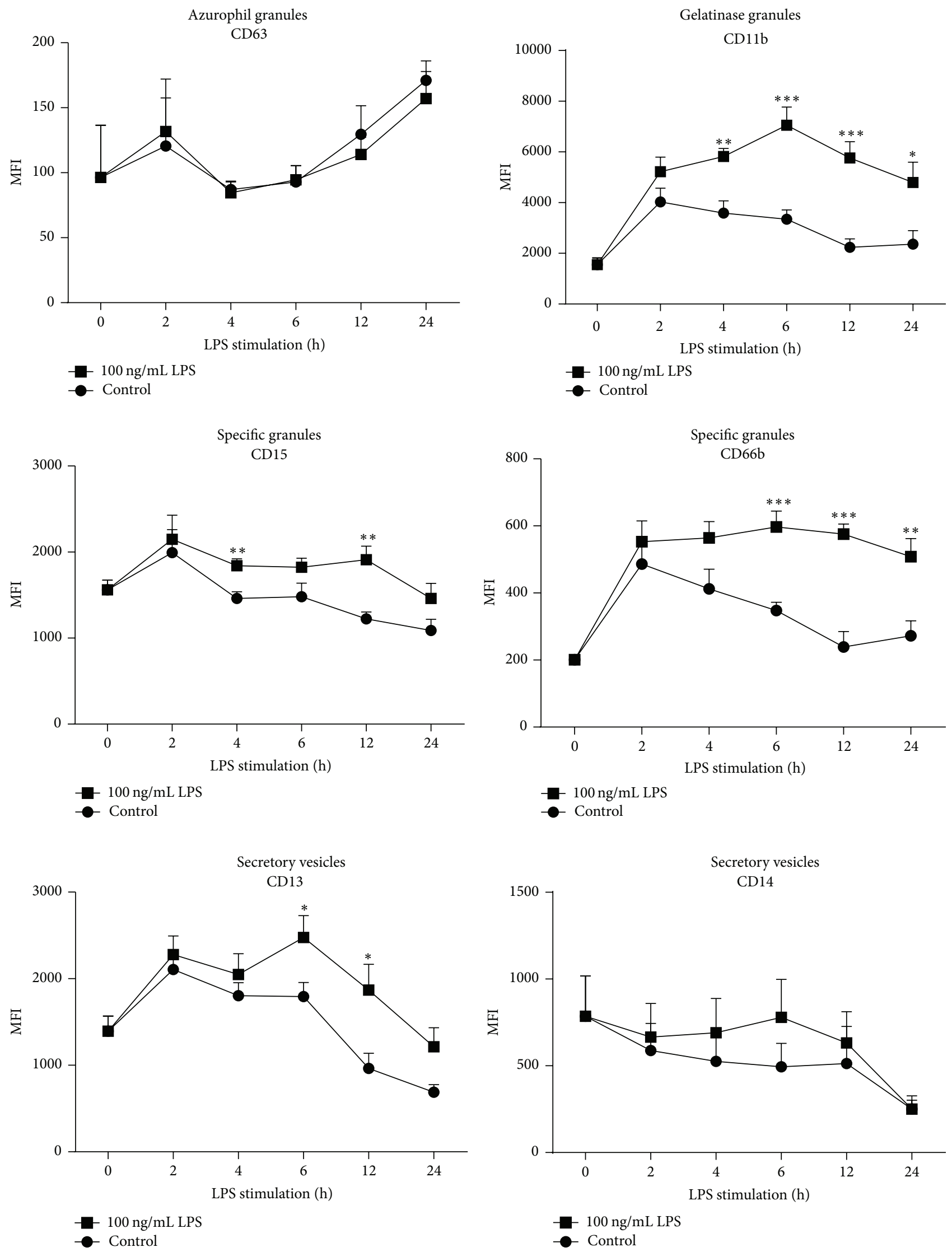

FIGURE 2: Continued. 

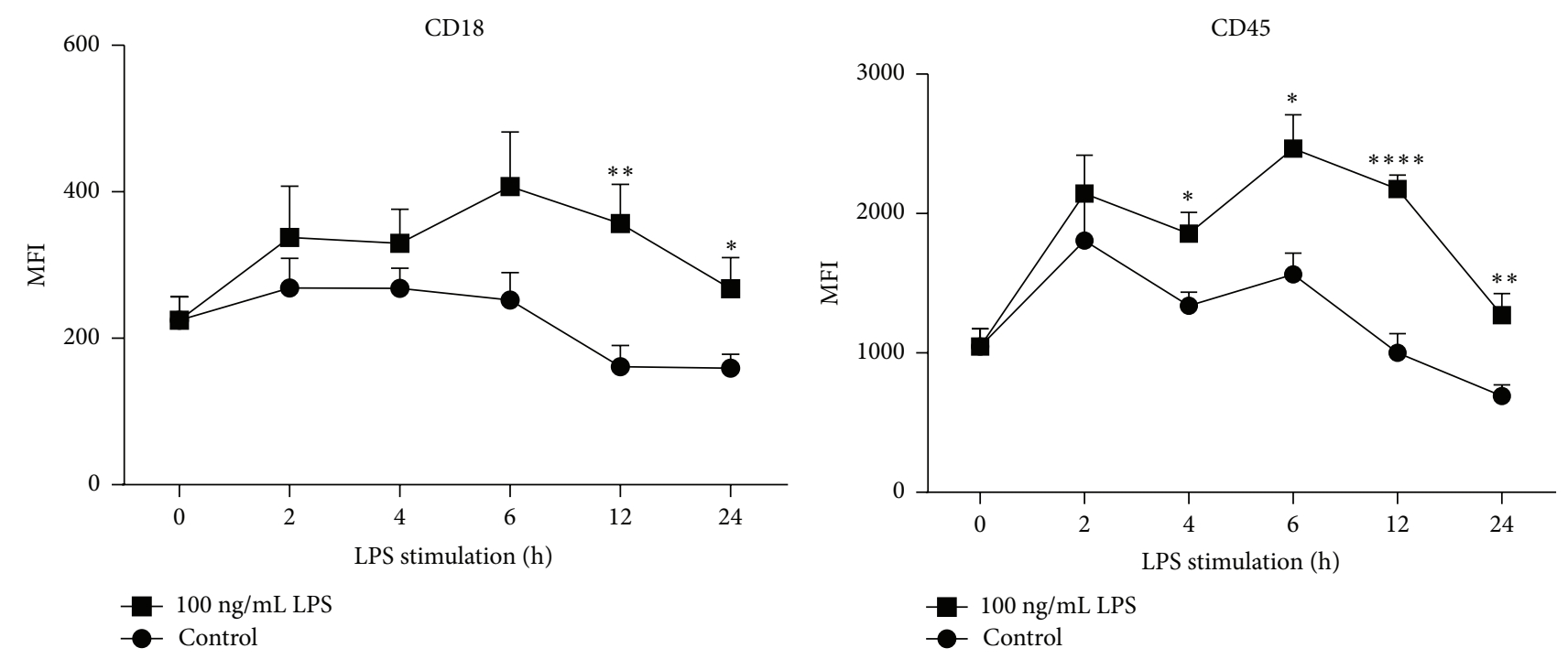

FIGURE 2: Temporal pattern of degranulation upon LPS stimulation in human neutrophils. Translocation of degranulation markers to the plasma membrane was assessed using flow cytometry after cell treatment with $100 \mathrm{ng} / \mathrm{mL}$ LPS for $0 \mathrm{~h}, 2 \mathrm{~h}, 4 \mathrm{~h}, 6 \mathrm{~h}, 12 \mathrm{~h}$, and $24 \mathrm{~h}$. Sytox Blue negative cells were gated to exclude dead cells from the analysis. Results are expressed in mean fluorescence intensity (MFI) of LPS-stimulated cells and nonstimulated control \pm SEM of at least 3 independent experiments, significantly different from nonstimulated control at the same time point: ${ }^{*} p<0.05,{ }^{* *} p<0.01,{ }^{* * *} p<0.001$, and ${ }^{* * * *} p<0.0001$

of experiments consisted in identifying the kinetic degranulation profile of the different granule types upon timedependent LPS stimulation. Degranulation can be determined by the upregulation of granule membrane molecules as a consequence of membrane fusion from granules with the plasma membrane [35]. Therefore, LPS-treated cells were analyzed for cell surface expression of several CD molecules known as degranulation markers.

Results showed that LPS stimulation affected the ease of mobilization of intracellular granule types in neutrophils in a time-dependent manner, as reflected by increased translocation of $\mathrm{CD}$ markers to the cell surface (Figure 2). In a temporal pattern, LPS stimulation induced the release of specific granules as demonstrated by the redistribution of CD15 and CD66b to the plasma membrane. Translocation of these $\mathrm{CD}$ markers towards the plasma membrane was scattered over a time interval of $4 \mathrm{~h}$ and $24 \mathrm{~h}$.

In a similar way, LPS stimulation increased the presence of CD11b as well as CD13, CD18, and CD45 at the plasma membrane reflecting an increased release of gelatinase granules and secretory vesicles, respectively. As observed with $\mathrm{CD} 15$ and CD66b for the specific granules, increase of these $\mathrm{CD}$ markers was detected between $4 \mathrm{~h}$ and $24 \mathrm{~h}$ of LPS stimulation.

Maximal expression at the plasma membrane for all the $\mathrm{CD}$ markers was detected after $6 \mathrm{~h}$ or $12 \mathrm{~h}$ of LPS stimulation (Figure 2).

It must be noted that LPS was unable to trigger the mobilization of azurophil granules since CD63 expression was not changed at the plasma membrane.

3.3. Linear Fitting of Cytokine Secretion and Degranulation Kinetics. Many approaches exist for the examination of time series of expression data (e.g., [36]) but none of them could be applied to analyze short-time series of secretion. For this reason, we used a novel model approach to explore the relationship between cytokine secretion and degranulation by their kinetic profiles (Figure 3).

We hypothesized that time series of secreted cytokines with similar pattern to time series of degranulation markers present at the plasma membrane should have proportional values at each time point of LPS stimulation, so that a proportionality factor between the two profile curves can be defined. To address this question, we choose to use the linear regression approach, which fits best to our needs: it captures proportionality well, can be used with only one pair of profiles (in our case, cytokine versus granule-specific marker), and includes measures to evaluate the results (significance of the model and $R$-squared value, Section 2). All ratio values between LPS-stimulated and nonstimulated control conditions from the time series of cytokine secretion and degranulation (Table 2) were $\log _{10}$ normalized.

While the ANOVA analyses the efficiency of this model (i.e., proportional kinetic profile curves are significantly different from the null model), the adjusted RSQ value measures the correlation between the kinetic profiles (i.e., proximity to the linear fitting). The optimal RSQ value was determined by simulations, in which predefined perturbations were introduced to our kinetic profiles (Section 2).

How augmenting perturbations ( $e$ from 0 to 1 ) influenced the linear fitting of two time series, in our example, the linear fitting between the granule marker CD11b and the cytokine IL8, is presented in Figure 4(a). The original kinetic profile is depicted by the black line whereas the one with perturbations is represented by the red line. By plotting the average RSQ values (derived from 1000 repetitions of simulations) against 
TABLE 2: Time-dependent (a) secretion of cytokines and (b) granules expressed in ratio between LPS-stimulated and nonstimulated cells \pm SEM of at least 3 independent experiments, significantly different from nonstimulated control at the same time point: ${ }^{*} p<0.05,{ }^{* *} p<0.01$, ${ }^{* * *} p<0.001$, and ${ }^{* * * *} p<0.0001$.

(a)

\begin{tabular}{|c|c|c|c|c|c|c|}
\hline Relative secretion & $0 \mathrm{~h}$ LPS & $2 \mathrm{~h} \mathrm{LPS}$ & $4 \mathrm{~h}$ LPS & $6 \mathrm{~h} \mathrm{LPS}$ & $12 \mathrm{~h} \mathrm{LPS}$ & $24 \mathrm{~h} \mathrm{LPS}$ \\
\hline $\mathrm{TNF} \alpha$ & $1.28 \pm 0.28$ & $6.30 \pm 1.81^{*}$ & $24.82 \pm 8.26$ & $51.54 \pm 14.79$ & $36.70 \pm 11.20^{* * *}$ & $16.48 \pm 11.35$ \\
\hline $\operatorname{IL} 1 \alpha$ & $0.73 \pm 0.38$ & $1.43 \pm 0.21$ & $3.36 \pm 1.92$ & $6.84 \pm 4.92$ & $6.67 \pm 3.51^{* * *}$ & $14.70 \pm 8.51^{*}$ \\
\hline $\operatorname{IL} 1 \beta$ & $0.69 \pm 0.69$ & $1.64 \pm 0.2^{*}$ & $4.87 \pm 2.55$ & $8.45 \pm 5.89$ & $7.89 \pm 2.3^{*}$ & $4.20 \pm 3.46$ \\
\hline IL6 & $0.93 \pm 0.18$ & $1.30 \pm 0.37$ & $34.35 \pm 23.38^{*}$ & $69.92 \pm 50.91^{*}$ & $185.53 \pm 62.99^{* * *}$ & $23.64 \pm 10.51^{*}$ \\
\hline $\operatorname{IL} 12 \beta$ & $1.02 \pm 0.06$ & $0.88 \pm 0.04$ & $1.06 \pm 0.09$ & $1.16 \pm 0.19$ & $6.20 \pm 3.57$ & $1.08 \pm 0.25$ \\
\hline CCL2 & $0.97 \pm 0.13$ & $0.86 \pm 0.17$ & $1.11 \pm 0.18$ & $1.17 \pm 0.36$ & $6.43 \pm 1.48^{* *}$ & $1.69 \pm 0.66$ \\
\hline CCL3 & $1.43 \pm 1.43$ & $6.72 \pm 0.98^{* *}$ & $24.35 \pm 4.51^{*}$ & $66.06 \pm 17.86^{*}$ & $65.98 \pm 24.41^{* * *}$ & $11.05 \pm 4.62$ \\
\hline CCL4 & $1.06 \pm 0.07$ & $2.65 \pm 0.76$ & $8.10 \pm 3.09^{*}$ & $11.69 \pm 5.84^{*}$ & $69.14 \pm 15.76^{* * *}$ & $1.22 \pm 0.72$ \\
\hline CCL5 & $0.88 \pm 0.05$ & $1.07 \pm 0.24$ & $1.04 \pm 0.34$ & $0.89 \pm 0.22$ & $0.96 \pm 0.18$ & $1.05 \pm 0.29$ \\
\hline IL8 & $1.02 \pm 0.12$ & $4.89 \pm 1.47^{*}$ & $9.85 \pm 2.31^{*}$ & $15.84 \pm 3.93^{*}$ & $31.83 \pm 10.15^{* * *}$ & $27.00 \pm 8.89^{*}$ \\
\hline
\end{tabular}

(b)

\begin{tabular}{|c|c|c|c|c|c|c|}
\hline Relative degranulation & $0 \mathrm{~h}$ LPS & $2 \mathrm{~h}$ LPS & $4 \mathrm{~h}$ LPS & $6 \mathrm{~h} \mathrm{LPS}$ & $12 \mathrm{~h} \mathrm{LPS}$ & $24 \mathrm{~h} \mathrm{LPS}$ \\
\hline CD63 & $1.00 \pm 0.42$ & $1.09 \pm 0.33$ & $0.97 \pm 0.10$ & $1.02 \pm 0.12$ & $0.88 \pm 0.10$ & $0.92 \pm 0.12$ \\
\hline CD15 & $1.00 \pm 0.07$ & $1.08 \pm 0.14$ & $1.26 \pm 0.06^{* *}$ & $1.23 \pm 0.07$ & $1.56 \pm 0.13^{* *}$ & $1.36 \pm 0.16$ \\
\hline CD66b & $1.00 \pm 0.06$ & $1.14 \pm 0.13$ & $1.37 \pm 0.12$ & $1.72 \pm 0.14^{* * *}$ & $2.41 \pm 0.13^{* * *}$ & $1.87 \pm 0.20^{* *}$ \\
\hline CD11b & $1.00 \pm 0.17$ & $1.29 \pm 0.14$ & $1.62 \pm 0.09^{* *}$ & $2.11 \pm 0.21^{* * *}$ & $2.57 \pm 0.29^{* * *}$ & $2.03 \pm 0.34^{*}$ \\
\hline CD13 & $1.00 \pm 0.13$ & $1.08 \pm 0.10$ & $1.14 \pm 0.13$ & $1.38 \pm 0.14^{*}$ & $1.94 \pm 0.31^{*}$ & $1.76 \pm 0.32$ \\
\hline CD14 & $1.00 \pm 0.30$ & $1.13 \pm 0.33$ & $1.31 \pm 0.37$ & $1.58 \pm 0.44$ & $1.23 \pm 0.35$ & $1.00 \pm 0.31$ \\
\hline CD18 & $1.00 \pm 0.14$ & $1.26 \pm 0.26$ & $1.23 \pm 0.17$ & $1.61 \pm 0.3$ & $2.22 \pm 0.33^{* *}$ & $1.68 \pm 0.27^{*}$ \\
\hline CD45 & $1.00 \pm 0.12$ & $1.19 \pm 0.15$ & $1.39 \pm 0.11^{*}$ & $1.58 \pm 0.16^{*}$ & $2.18 \pm 0.10^{* * * *}$ & $1.84 \pm 0.22^{* *}$ \\
\hline
\end{tabular}

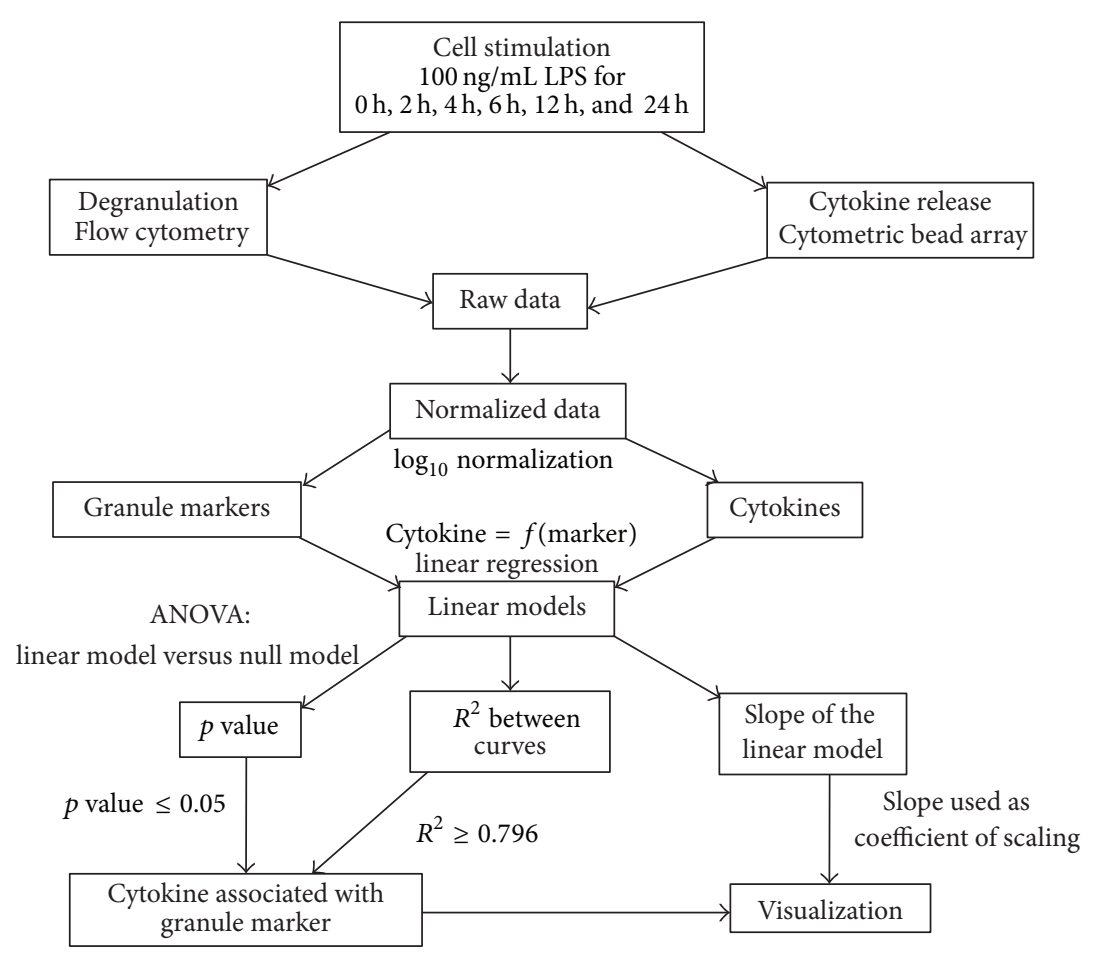

FIGURE 3: Workflow of linear fitting of LPS-mediated cytokine secretion and degranulation. A novel approach based on linear fitting was used to find linear relationship between short-time series of secreted cytokines and similar pattern to degranulation markers present at the plasma membrane. After importing data into R statistical software, all ratio values between LPS-stimulated and nonstimulated control conditions were $\log _{10}$ normalized and treated as mentioned in Section 2. 

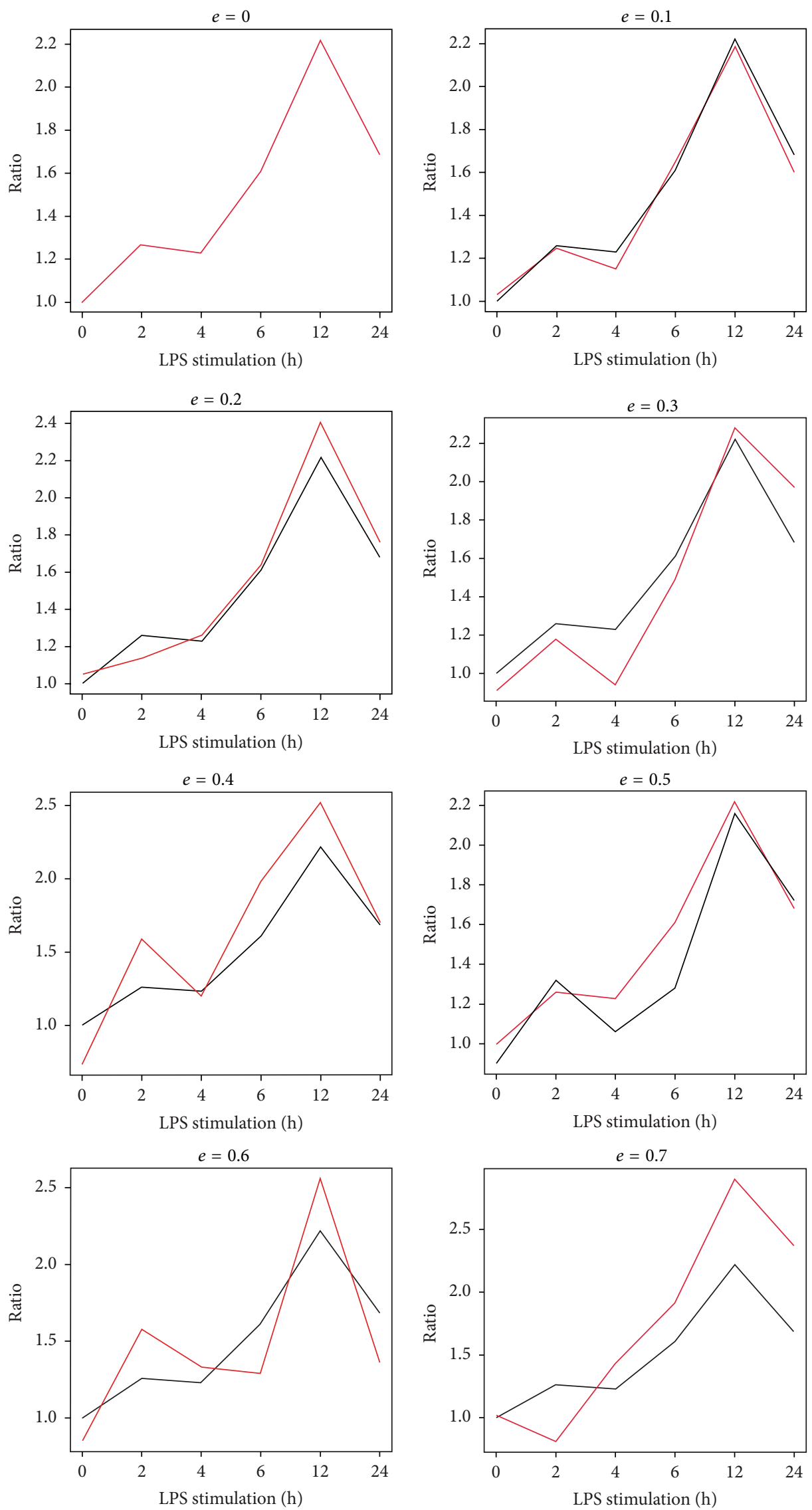

Figure 4: Continued. 

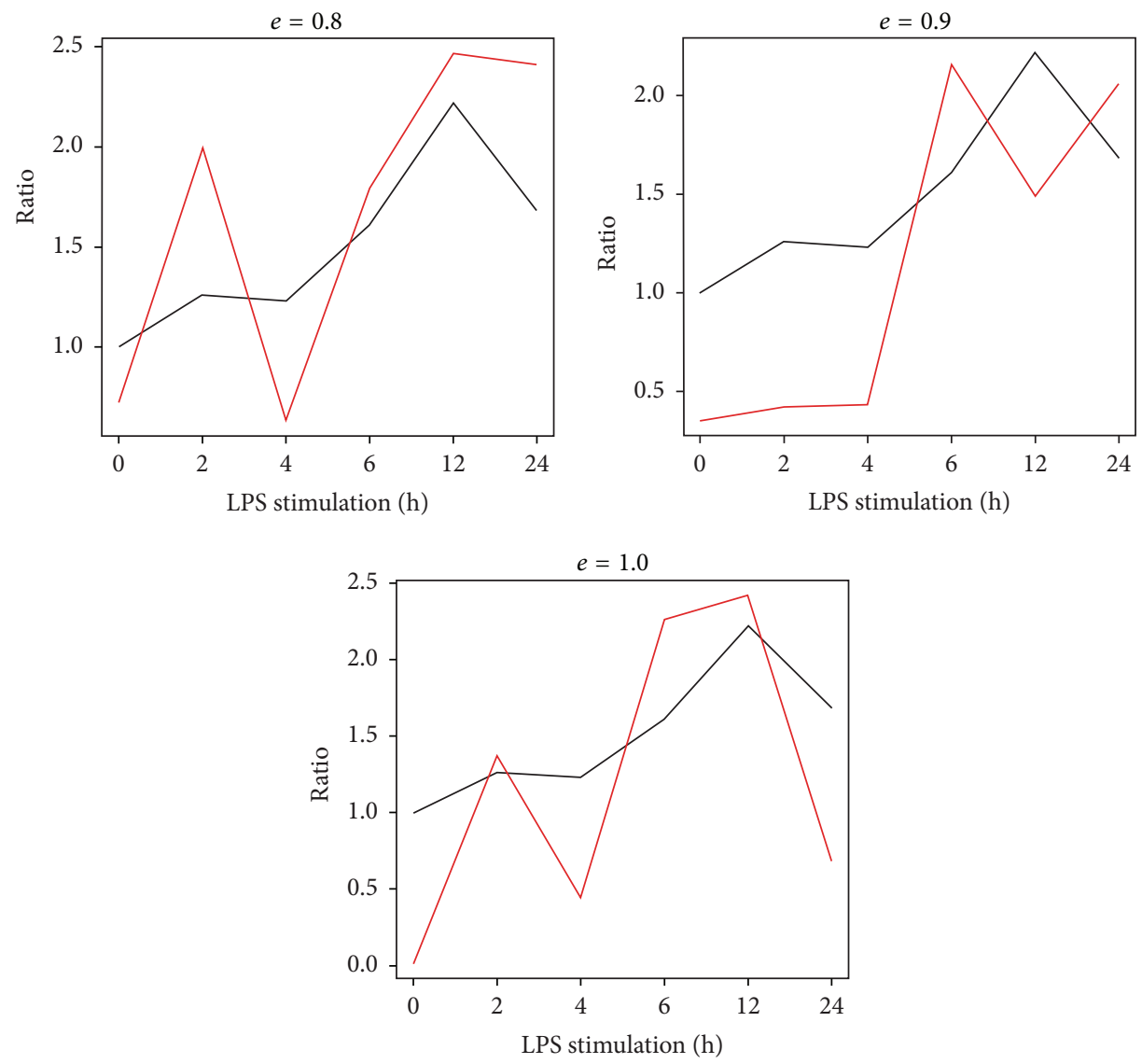

(a)

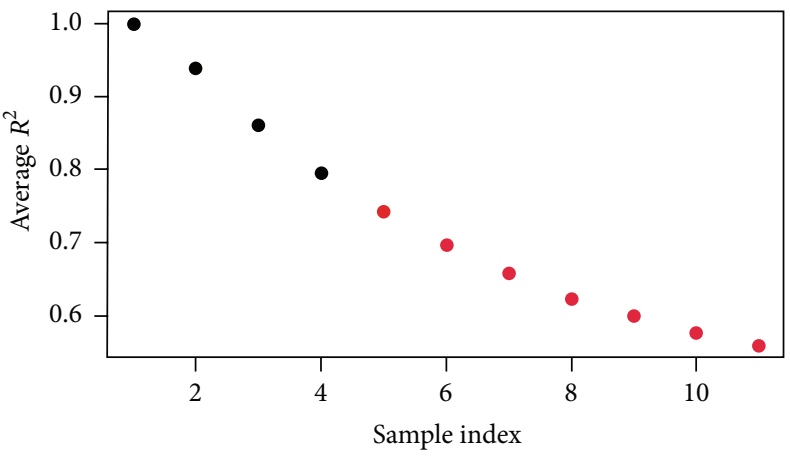

(b)

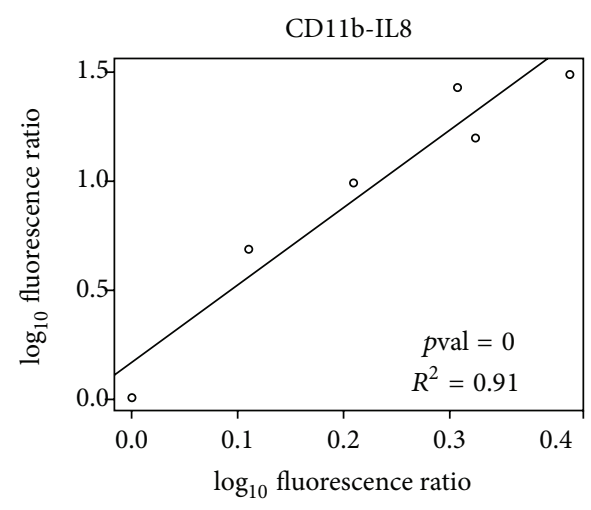

(c)

FIGURE 4: Linear fitting approach to investigate time series of degranulation and cytokine secretion. (a) Simulations were performed to find the optimal threshold for RSQ value. After introducing perturbations ( $e$ value) to the kinetic profiles, the linear fitting decreases in significance. (b) Plot of average RSQ deriving from simulations against augmenting $e$ values. (c) Perfectly linear fitting model in which the behaviour of IL8 is correlated to CD11b. Due to its $p$ value of 0 and RSQ of 0.91 , the correlation between IL8 and CD11b corresponds to a perfect linear fitting model.

$e$ values, $k$-means clustering can differentiate between "high" (black) and "low" (red) RSQ (Figure 4(b)).

By setting these parameters nonsignificant outcomes with "low" RSQ were eliminated, and the threshold for RSQ was set to $R S Q \geq 0.796$.

An example of a significant linear fitting model is shown (Figure 4(c)), in which the behaviour of IL8 is correlated to
CD11b. Due to its $p$ value of 0 and RSQ of 0.91 , the correlation between IL8 and CD11b fits to the model.

\subsection{Relationship between Degranulation and Cytokine Secre-} tion. After filtering only highly significant correlation data, results from human neutrophils were plotted. The granule membrane molecules (CD63, CD66b, CD11b, and CD45), 


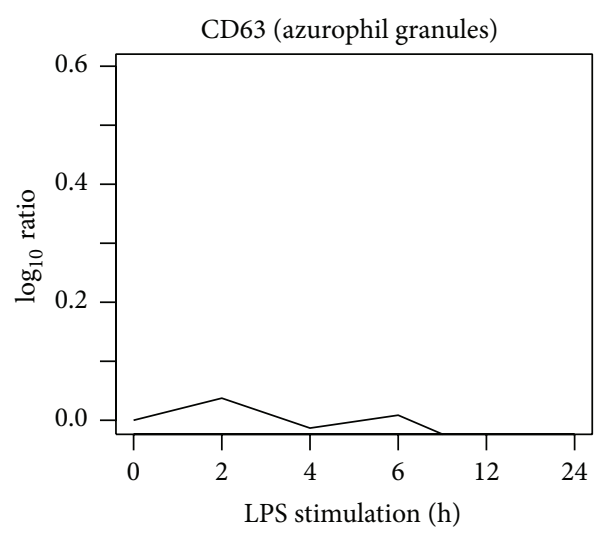

- CD63

(a)

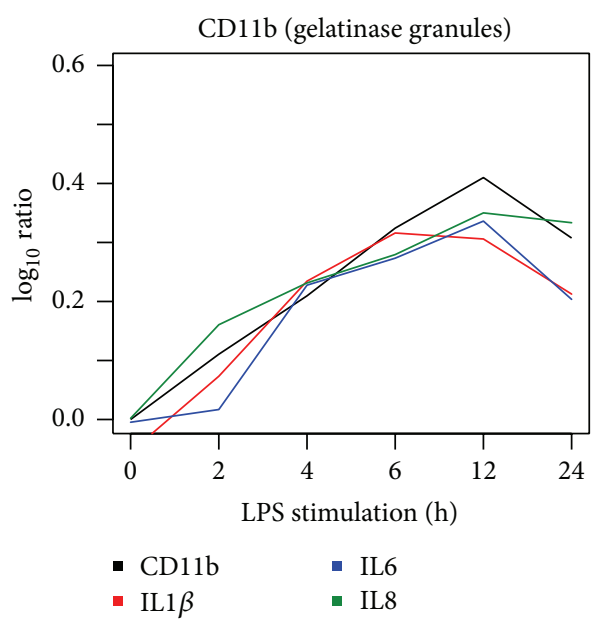

(c)

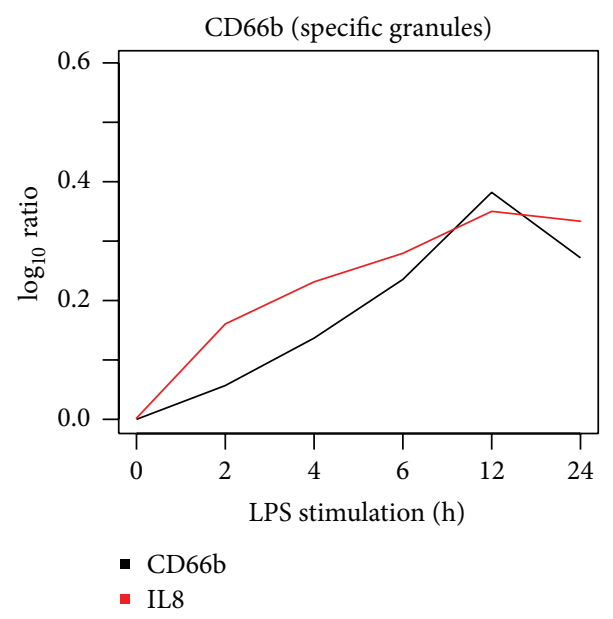

(b)

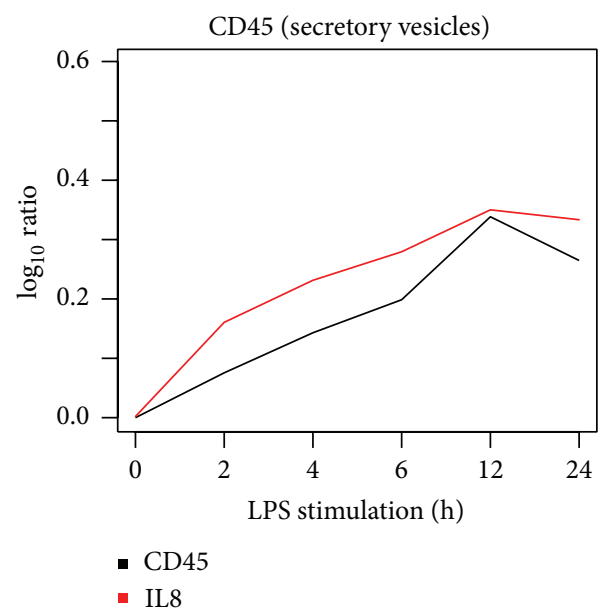

(d)

FIGURE 5: Linear fitting of LPS-mediated kinetics of cytokine release and degranulation in human neutrophils. Enlisted cytokines on the histograms fitted to the kinetics of the degranulation markers characteristic for (a) azurophil granules (CD63), (b) specific granules (CD66b), (c) gelatinase granules (CD11b), and (d) secretory vesicles (CD45), according to the selection of $p$ value $\leq 0.05$ and RSQ $\geq 0.82$.

specific of each type of granule, that are most highly upregulated have been targeted to show the linear fitting approach (Figure 5).

This method applied to the time series data showed that the secretion of three selected proinflammatory cytokines (IL8, IL6, and IL1 $\beta$ ) strongly correlated with the release of secretory vesicles, gelatinase granules, and specific granules (Figure 5).

The release of the cytokine IL8 fitted to CD66b suggesting that secretion of this cytokine correlated to specific granules (Figure 5(b)). Moreover, time series of IL1 $\beta$, IL8, and IL6 release were strongly correlated to the degranulation marker CD11b, showing a relationship between gelatinase granules and these cytokines (Figure 5(c)).

Furthermore, secretory vesicles represented by the marker CD45 were fitted to IL8 (Figure 5(d)).

Since no significant cytokine correlation has been observed for CD63, azurophil granules are probably not associated with cytokine secretion (Figure 5(a)).

\section{Discussion}

For many years, the contributory impact of neutrophils to the development of chronic inflammation was not seriously taken into account since they have been considered as terminally differentiated cells synthesizing low amount of RNA and protein [37]. However, the vast number of neutrophils found at the site of infection cannot be neglected due to the fact that their secreted amounts of granule proteins and cytokines exert a cumulative and synergistic effect on the inflammatory tissue environment [15]. These proinflammatory soluble mediators are highly decisive for the onset of inflammatory processes and the activation and the recruitment of various immune cells to the infection site [1]. However, little is known about the combination in which cytokines and granule proteins are secreted by neutrophils. In the present report, we therefore aimed to predict the spatiotemporal regulation of proinflammatory mediator release in neutrophils. For this purpose, LPS has been used as stimulus agent since it has been 
well described to induce the secretion of granule contents and cytokines [17, 38]. Once the model is established, it could constitute an important tool to investigate other stimulation conditions (fMLF, TNF $\alpha$, IL8, or combination of stimuli) in order to mimic different microenvironmental conditions (e.g., healthy and pathological diseases) and help to improve our knowledge of inflammatory processes.

Our study is the first to propose an original approach allowing the establishment of a relationship between cytokine secretion and degranulation in neutrophils. We choose to use the linear fitting approach to integrate data generated from own experiments and obtained from LPS-mediated shorttime series of degranulation and cytokine secretion. Other approaches, such as Pearson correlation, are based only on the average of all values correlated. In contrast, our model is able to reliably predict time-specific associations between the two dynamic functions in neutrophils, respecting each time point of stimulation. According to our results, a number of cytokines could be fitted to the different types of neutrophil granules. These granules have been characterized to be mobilized towards the plasma membrane in a hierarchically and more precisely reverse order to their formation according to the formed-first-released-last model [2]. Our model illustrates the fact that neutrophil-derived cytokines and granules are released in a hierarchical sequence in accordance with their roles during the microbial elimination processes and inflammatory response (Figure 6(a)).

In this study, the linear fitting approach (i) gives us information about the concurrent behavior of cytokine secretion and degranulation upon inflammation, thus underlining the key role of both functions in the regulation of inflammatory responses and (ii) can represent an attractive method to investigate the possible mobilization or localization of cytokines in the different types of granules.

Given that cytokines can exert pleiotropic functions, some of them are probably localized in different types of granules as suggested by our model. In this sense, we found that IL8 correlated to secretory vesicles (Figure 6(a)), which are the most easily mobilized organelles in mature neutrophils [35]. Furthermore, IL8 was correlated to gelatinase and specific granules. These data support the observations of Pellmé et al. [2], who reported that IL8 is stored in cytoplasmic granules in resting peripheral blood neutrophils and, thus, can be rapidly mobilized and released by the cells.

IL8 can be secreted into the extracellular milieu from intracellular stores or by de novo synthesis via the classical secretory pathway. Two phases of secretion have been described: an early secretory phase which is directly induced by LPS and a late secretory phase which results from LPSstimulated release of other proinflammatory mediators such as TNF $\alpha$ and IL1 $\beta$ [39]. The fact that IL 8 is stored in different types of granules could allow its secretion over a large time interval.

Large amount of IL8 released could be explained by a positive feedback loop generated by MMP-9 on the IL8induced neutrophil activity. Indeed, it has been previously reported that MMP-9 released from gelatinase granules is able to process IL8 [40] which is stored in the same granules as MMP-9 before secretion as shown by our linear fitting approach. IL8 cleaved by MMP-9 can enhance neutrophil degranulation [40], in comparison to nondegraded IL8, and thus increase the quantity of MMP-9 and IL8 released leading to an amplification of this system.

Our results also show a significant fitting between kinetics of IL6 secretion and the release of gelatinase granules. In line with this observation, Terebuh et al. [41] showed by immunohistochemical staining of neutrophils that IL6 might be localized in gelatinase granules and secretory vesicles.

Finally, in our model, IL1 $\beta$ could be fitted to gelatinase degranulation as previously postulated [17]. IL1 $\beta$ is secreted by a nonclassical secretory pathway (independent of endoplasmic reticulum and Golgi apparatus). Different release models have been suggested for IL1 $\beta$ [42] but the mechanisms associated are poorly understood and still controversially discussed. Our data indicate that the process of IL1 $\beta$ secretion involves a trafficking via granules which could be related to gelatinase granules.

In contrast to other granules, degranulation of azurophil granules seems unchanged by LPS suggesting that either (i) this granule type may require further cell activation to induce its mobilization towards the plasma membrane [43] or (ii) the upregulation of CD63 at the plasma membrane might not be significant enough to detect since azurophil granules are rather poor in receptors in contrast to secretory vesicles [35]. For this reason, no cytokine could probably be fitted to CD63 in human neutrophils.

In this view, late release of azurophil granule contents can be explained by the involvement of these proteins in neutrophil extracellular trap formation [43]. In this regard, the role of these granules during cytokine secretion appears very restricted. This assumption is supported by our results showing that azurophil granules are not able to translocate to the plasma membrane upon LPS stimulation in neutrophils.

\section{Conclusion}

Intracellular localization of cytokines in neutrophils remains largely elusive due to the fact that reliable staining for electron microscopy is facing challenges as the low intracellular amount of cytokines and other techniques used to document subcellular organelle location of cytokines have limited resolution (e.g., subcellular fractionation) [44].

In comparison to the modelling approach proposed by Rørvig et al. [45], which is based on proteomic and mRNA array data to predict localization of proteins in granules, our approach is complementary by including functional data analysis. Hence, the linear fitting between degranulation and cytokine release in LPS-treated neutrophils represents an attractive method to investigate the possible localization of cytokines in the different types of granules even if additional experiments are required to confirm the intracellular localization of cytokines. Furthermore, since our linear fitting approach has been adapted to investigate secretion kinetics, it can easily be extrapolated for the analysis of other short-time series deriving from other cell types, disease, or developmental states, for example, protein arrays or proteomics data.

Our linear fitting approach primarily constitutes a tool aiming to investigate regulatory mechanisms during 


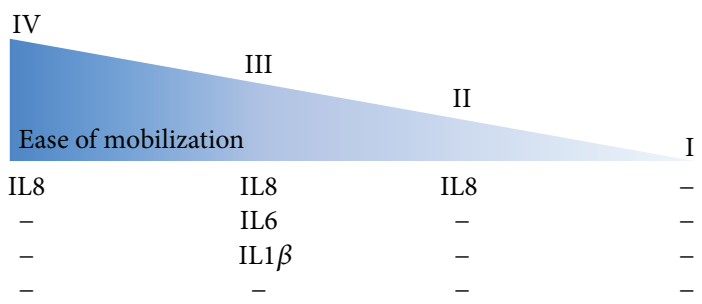

(a)

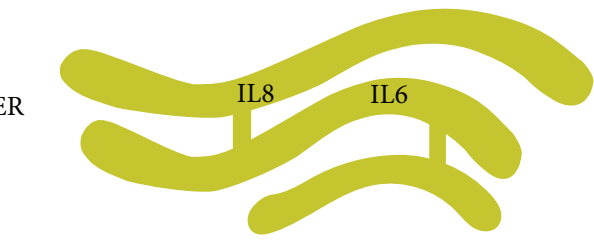

Cytosol
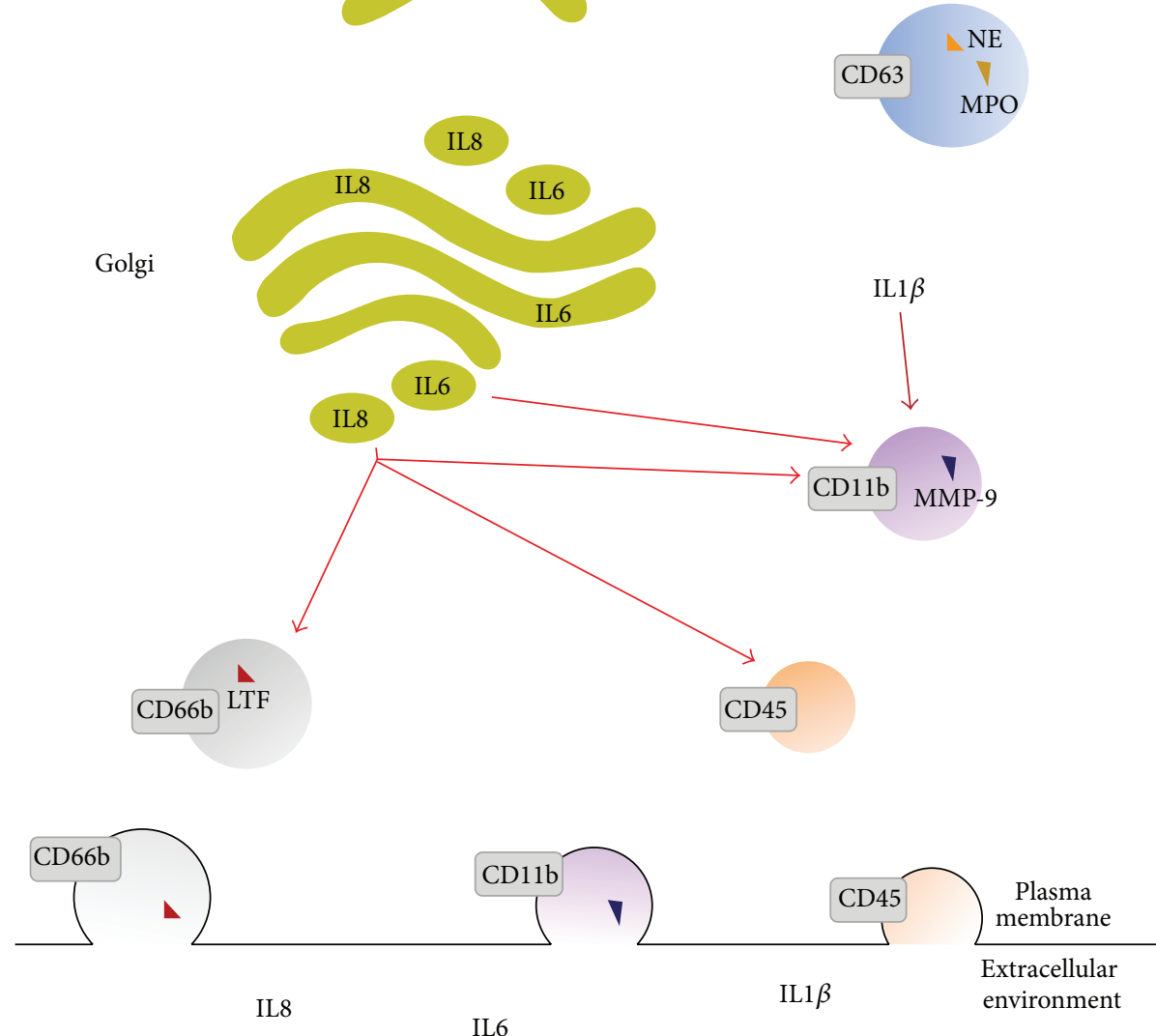

(b)

Figure 6: Hypothetical models of fitted cytokines and granules in human neutrophils. (a) Neutrophil-derived cytokines are released in a hierarchical sequence coincident with the roles of granules (IV secretory vesicles, III gelatinase granules, II specific granules, and I azurophil granules) during the microbial elimination processes and inflammatory response. (b) Cytokines and granules are released in a concurrent fashion but could additionally be localized in or mobilized to the different granule types. Classical secretory pathways are mediated through the endoplasmic reticulum (ER) and Golgi complex (IL6 and IL8). IL1 $\beta$ is secreted on a nonconventional pathway. Possible routes for cytokine trafficking (after ER-Golgi or after cleavage) and mobilization to the plasma membrane relative to degranulation are shown in red. Different types of granules are characterized by their CD markers and proteolytic enzymes (triangles): CD63, myeloperoxidase (MPO), and neutrophil elastase (NE) for azurophil granules; CD66b and lactoferrin (LTF) for specific granules; CD11b and matrix metallopeptidase-9 (MMP-9) for gelatinase granules; and CD45 for secretory vesicles. Upon mobilization of the granules to the plasma membrane, granule docking and fusion lead to the translocation of the CD markers to the plasma membrane and the release of proteolytic enzymes. 
neutrophil exocytosis but can also serve as basis to identify regulatory proteins by the supplementary analysis of proteins involved in exocytosis (e.g., SNARE and Rab proteins) and the construction of a dynamic regulatory network [46].

\section{Abbreviations \\ CBA: Cytometric bead array \\ LPS: Bacterial lipopolysaccharide.}

\section{Conflict of Interests}

The authors declare that there is no conflict of interests regarding the publication of this paper.

\section{Authors' Contribution}

Isabelle Naegelen and Nicolas Beaume contributed equally to this paper.

\section{Acknowledgments}

The authors thank the healthy volunteers for their blood donation, the Croix-Rouge Luxembourgeoise, and the Centre Hospitalier du Luxembourg for their collaboration. Financial support was provided by the University of Luxembourg.

\section{References}

[1] C. Nathan, "Neutrophils and immunity: challenges and opportunities," Nature Reviews Immunology, vol. 6, no. 3, pp. 173-182, 2006.

[2] S. Pellmé, M. Mörgelin, H. Tapper, U.-H. Mellqvist, C. Dahlgren, and A. Karlsson, "Localization of human neutrophil interleukin-8 (CXCL-8) to organelle(s) distinct from the classical granules and secretory vesicles," Journal of Leukocyte Biology, vol. 79, no. 3, pp. 564-573, 2006.

[3] M. A. Cassatella, "The production of cytokines by polymorphonuclear neutrophils," Immunology Today, vol. 16, no. 1, pp. 21-26, 1995.

[4] P. Scapini, J. A. Lapinet-Vera, S. Gasperini, F. Calzetti, F. Bazzoni, and M. A. Cassatella, "The neutrophil as a cellular source of chemokines," Immunological Reviews, vol. 177, pp. 195-203, 2000.

[5] T. Kasama, Y. Miwa, T. Isozaki, T. Odai, M. Adachi, and S. L. Kunkel, "Neutrophil-derived cytokines: potential therapeutic targets in inflammation," Current Drug Targets: Inflammation and Allergy, vol. 4, no. 3, pp. 273-279, 2005.

[6] A. C. Stanley and P. Lacy, "Pathways for cytokine secretion," Physiology, vol. 25, no. 4, pp. 218-229, 2010.

[7] E. H. Duitman, Z. Orinska, and S. Bulfone-Paus, "Mechanisms of cytokine secretion: a portfolio of distinct pathways allows flexibility in cytokine activity," European Journal of Cell Biology, vol. 90, no. 6-7, pp. 476-483, 2011.

[8] E. Crivellato, B. Nico, F. Mallardi, C. A. Beltrami, and D. Ribatti, "Piecemeal degranulation as a general secretory mechanism?" Anatomical Record Part A: Discoveries in Molecular, Cellular, and Evolutionary Biology, vol. 274, no. 1, pp. 778-784, 2003.

[9] R. Moqbel and J. J. Coughlin, "Differential secretion of cytokines," Science Signaling, vol. 2006, no. 338, article pe26, 2006.
[10] J. A. Pickett and J. M. Edwardson, "Compound exocytosis: mechanisms and functional significance," Traffic, vol. 7, no. 2, pp. 109-116, 2006.

[11] A. Matsukawa and M. Yoshinaga, "Sequential generation of cytokines during the initiative phase of inflammation, with reference to neutrophils," Inflammation Research, vol. 47, supplement 3, pp. S137-S144, 1998.

[12] G. Radford-Smith and D. P. Jewell, "Cytokines and inflammatory bowel disease," Bailliere's Clinical Gastroenterology, vol. 10, no. 1, pp. 151-164, 1996.

[13] S. W. Edwards and M. B. Hallett, "Seeing the wood for the trees: the forgotten role of neutrophils in rheumatoid arthritis," Immunology Today, vol. 18, no. 7, pp. 320-324, 1997.

[14] W. Q. Gan, S. F. P. Man, A. Senthilselvan, and D. D. Sin, "Association between chronic obstructive pulmonary disease and systemic inflammation: a systematic review and a metaanalysis," Thorax, vol. 59, no. 7, pp. 574-580, 2004.

[15] O. Soehnlein, "Multiple roles for neutrophils in atherosclerosis," Circulation Research, vol. 110, no. 6, pp. 875-888, 2012.

[16] T. W. Kuijpers, A. T. J. Tool, C. E. Van der Schoot et al., "Membrane surface antigen expression on neutrophils: a reappraisal of the use of surface markers for neutrophil activation," Blood, vol. 78, no. 4, pp. 1105-1111, 1991.

[17] I. Naegelen, S. Plançon, N. Nicot et al., "An essential role of syntaxin 3 protein for granule exocytosis and secretion of IL$1 \alpha$, IL-1 $\beta$, IL-12b, and CCL4 from differentiated HL-60 cells," Journal of Leukocyte Biology, vol. 97, no. 3, pp. 557-571, 2015.

[18] T. Billiet, P. Rutgeerts, M. Ferrante, G. Van Assche, and S. Vermeire, "Targeting TNF-alpha for the treatment of inflammatory bowel disease," Expert Opinion on Biological Therapy, vol. 14, no. 1, pp. 75-101, 2014.

[19] Y. Zhang, X. Yang, F. Bian et al., “TNF- $\alpha$ promotes early atherosclerosis by increasing transcytosis of LDL across endothelial cells: crosstalk between NF- $\kappa$ B and PPAR- $\gamma$," Journal of Molecular and Cellular Cardiology, vol. 72, pp. 85-94, 2014.

[20] Y. Li, Z. Zhang, Y. Xu, S. Xiong, W. Ni, and S. Chen, “TNF- $\alpha$ upregulates matrix metalloproteinase- 9 expression and activity in alveolar macrophages from patients with chronic obstructive pulmonary disease," Journal of Huazhong University of Science and Technology, vol. 26, no. 6, pp. 647-650, 2006.

[21] W. Hueber, B. H. Tomooka, X. Zhao et al., "Proteomic analysis of secreted proteins in early rheumatoid arthritis: anti-citrulline autoreactivity is associated with up regulation of proinflammatory cytokines," Annals of the Rheumatic Diseases, vol. 66, no. 6, pp. 712-719, 2007.

[22] O. Ludwiczek, E. Vannier, I. Borggraefe et al., "Imbalance between interleukin-1 agonists and antagonists: relationship to severity of inflammatory bowel disease," Clinical and Experimental Immunology, vol. 138, no. 2, pp. 323-329, 2004.

[23] J. Hartman and W. H. Frishman, "Inflammation and atherosclerosis: a review of the role of interleukin- 6 in the development of atherosclerosis and the potential for targeted drug therapy," Cardiology in Review, vol. 22, no. 3, pp. 147-151, 2014.

[24] R. Garrod, J. Marshall, E. Barley, S. Fredericks, and G. Hagan, "The relationship between inflammatory markers and disability in chronic obstructive pulmonary disease (COPD)," Primary Care Respiratory Journal, vol. 16, no. 4, pp. 236-240, 2007.

[25] K. Vandenbroeck, I. Alloza, M. Gadina, and P. Matthys, "Inhibiting cytokines of the interleukin-12 family: recent advances and novel challenges," Journal of Pharmacy and Pharmacology, vol. 56, no. 2, pp. 145-160, 2004. 
[26] C. Schmidt, T. Marth, B. M. Wittig, A. Hombach, H. Abken, and A. Stallmach, "Interleukin-12 antagonists as new therapeutic agents in inflammatory bowel disease," Pathobiology, vol. 70, no. 3, pp. 177-183, 2003.

[27] K. Yong, G. Dogra, N. Boudville et al., "Interleukin-12 is associated with arterial stiffness in healthy individuals," American Journal of Hypertension, vol. 26, no. 2, pp. 159-162, 2013.

[28] R. E. Gerszten, E. A. Garcia-Zepeda, Y.-C. Lim et al., "MCP1 and IL-8 trigger firm adhesion of monocytes to vascular endothelium under flow conditions," Nature, vol. 398, no. 6729, pp. 718-725, 1999.

[29] S. R. Chintalacharuvu, J. X. Wang, J. M. Giaconia, and C. Venkataraman, "An essential role for CCL3 in the development of collagen antibody-induced arthritis," Immunology Letters, vol. 100, no. 2, pp. 202-204, 2005.

[30] W. A. Kuziel, T. C. Dawson, M. Quinones et al., "CCR5 deficiency is not protective in the early stages of atherogenesis in apoE knockout mice," Atherosclerosis, vol. 167, no. 1, pp. 25-32, 2003.

[31] E. Robinson, E. C. Keystone, T. J. Schall, N. Gillett, and E. N. Fish, "Chemokine expression in rheumatoid arthritis (RA): evidence of RANTES and macrophage inflammatory protein (MIP)-1 $\beta$ production by synovial T cells," Clinical \& Experimental Immunology, vol. 101, no. 3, pp. 398-407, 1995.

[32] C. Banks, A. Bateman, R. Payne, P. Johnson, and N. Sheron, "Chemokine expression in IBD. Mucosal chemokine expression is unselectively increased in both ulcerative colitis and Crohn's disease," Journal of Pathology, vol. 199, no. 1, pp. 28-35, 2003.

[33] T.-C. Yao, M.-L. Kuo, L.-C. See et al., "RANTES and monocyte chemoattractant protein 1 as sensitive markers of disease activity in patients with juvenile rheumatoid arthritis: a six-year longitudinal study," Arthritis and Rheumatism, vol. 54, no. 8, pp. 2585-2593, 2006.

[34] K. Mitsuyama, A. Toyonaga, E. Sasaki et al., "IL-8 as an important chemoattractant for neutrophils in ulcerative colitis and Crohn's disease," Clinical and Experimental Immunology, vol. 96, no. 3, pp. 432-436, 1994.

[35] N. Borregaard and J. B. Cowland, "Granules of the human neutrophilic polymorphonuclear leukocyte," Blood, vol. 89, no. 10, pp. 3503-3521, 1997.

[36] J. Ernst and Z. Bar-Joseph, "STEM: a tool for the analysis of short time series gene expression data," BMC Bioinformatics, vol. 7, article 191, 2006.

[37] V. Witko-Sarsat, P. Rieu, B. Descamps-Latscha, P. Lesavre, and L. Halbwachs-Mecarelli, "Neutrophils: molecules, functions and pathophysiological aspects," Laboratory Investigation, vol. 80, no. 5, pp. 617-653, 2000.

[38] K. C. Malcolm and G. S. Worthen, "Lipopolysaccharide stimulates p38-dependent induction of antiviral genes in neutrophils independently of paracrine factors," The Journal of Biological Chemistry, vol. 278, no. 18, pp. 15693-15701, 2003.

[39] M. A. Cassatella, L. Meda, S. Bonora, M. Ceska, and G. Constantin, "Interleukin 10 (IL-10) inhibits the release of proinflammatory cytokines from human polymorphonuclear leukocytes. Evidence for an autocrine role of tumor necrosis factor and IL- $1 \beta$ in mediating the production of IL- 8 triggered by lipopolysaccharide," Journal of Experimental Medicine, vol. 178, no. 6, pp. 2207-2211, 1993.

[40] P. E. Van den Steen, P. Proost, A. Wuyts, J. Van Damme, and G. Opdenakker, "Neutrophil gelatinase B potentiates interleukin8 tenfold by aminoterminal processing, whereas it degrades
CTAP-III, PF-4, and GRO- $\alpha$ and leaves RANTES and MCP-2 intact," Blood, vol. 96, no. 8, pp. 2673-2681, 2000.

[41] P. D. Terebuh, I. G. Otterness, R. M. Strieter et al., "Biologic and immunohistochemical analysis of interleukin- 6 expression in vivo. Constitutive and induced expression in murine polymorphonuclear and mononuclear phagocytes," The American Journal of Pathology, vol. 140, no. 3, pp. 649-657, 1992.

[42] C. Eder, "Mechanisms of interleukin-1 $\beta$ release," Immunobiology, vol. 214, no. 7, pp. 543-553, 2009.

[43] K. D. Metzler, T. A. Fuchs, W. M. Nauseef et al., "Myeloperoxidase is required for neutrophil extracellular trap formation: implications for innate immunity," Blood, vol. 117, no. 3, pp. 953959, 2011.

[44] C. Pasquali, I. Fialka, and L. A. Huber, "Subcellular fractionation, electromigration analysis and mapping of organelles," Journal of Chromatography B: Biomedical Sciences and Applications, vol. 722, no. 1-2, pp. 89-102, 1999.

[45] S. Rørvig, O. Østergaard, N. H. H. Heegaard, and N. Borregaard, "Proteome profiling of human neutrophil granule subsets, secretory vesicles, and cell membrane: correlation with transcriptome profiling of neutrophil precursors," Journal of Leukocyte Biology, vol. 94, no. 4, pp. 711-721, 2013.

[46] B. A. Kidd, L. A. Peters, E. E. Schadt, and J. T. Dudley, "Unifying immunology with informatics and multiscale biology," Nature Immunology, vol. 15, no. 2, pp. 118-127, 2014. 


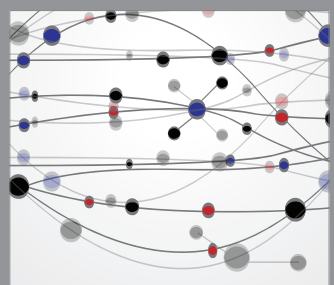

The Scientific World Journal
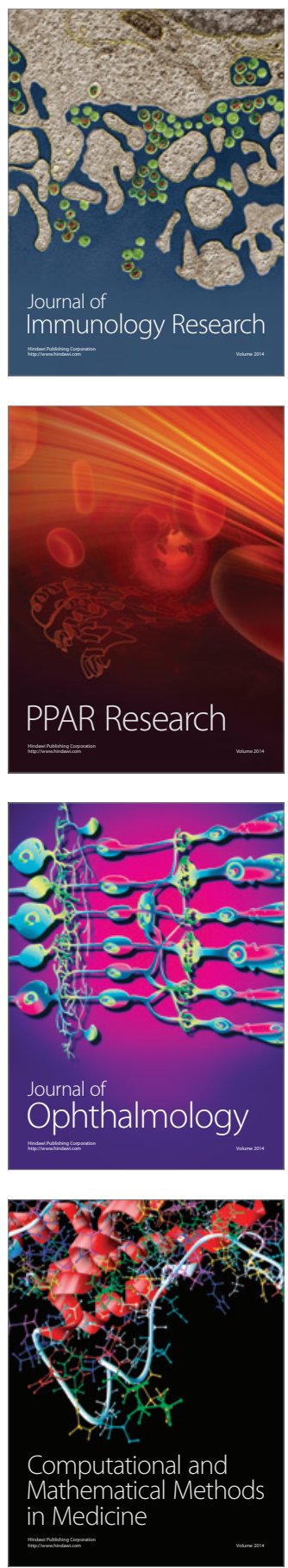

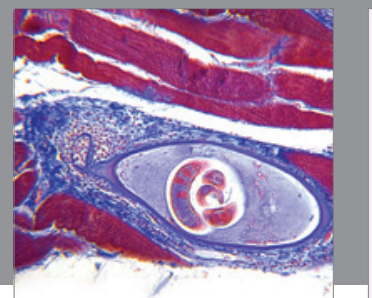

Gastroenterology

Research and Practice
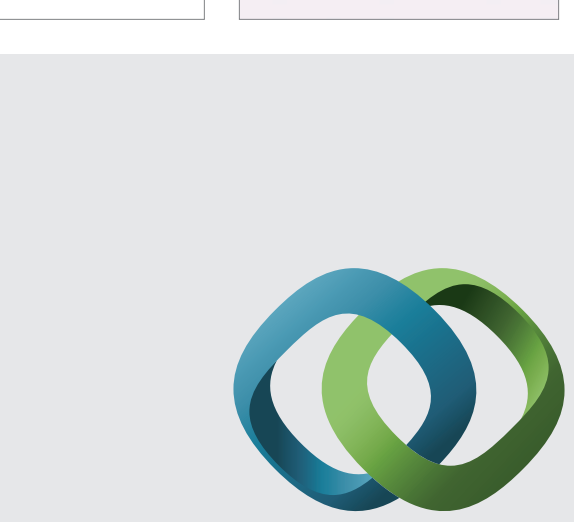

\section{Hindawi}

Submit your manuscripts at

http://www.hindawi.com
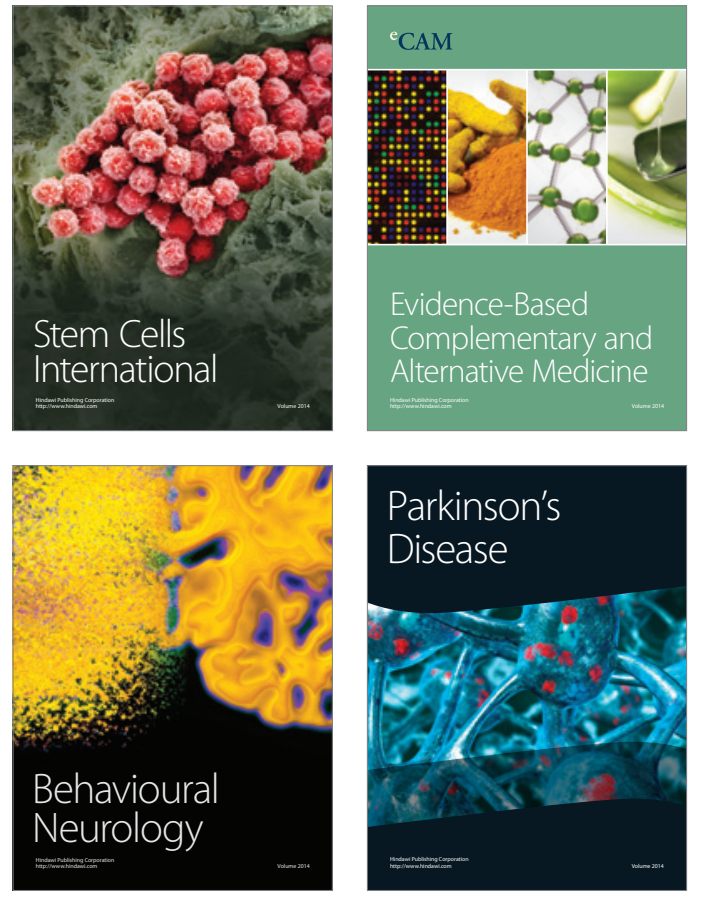
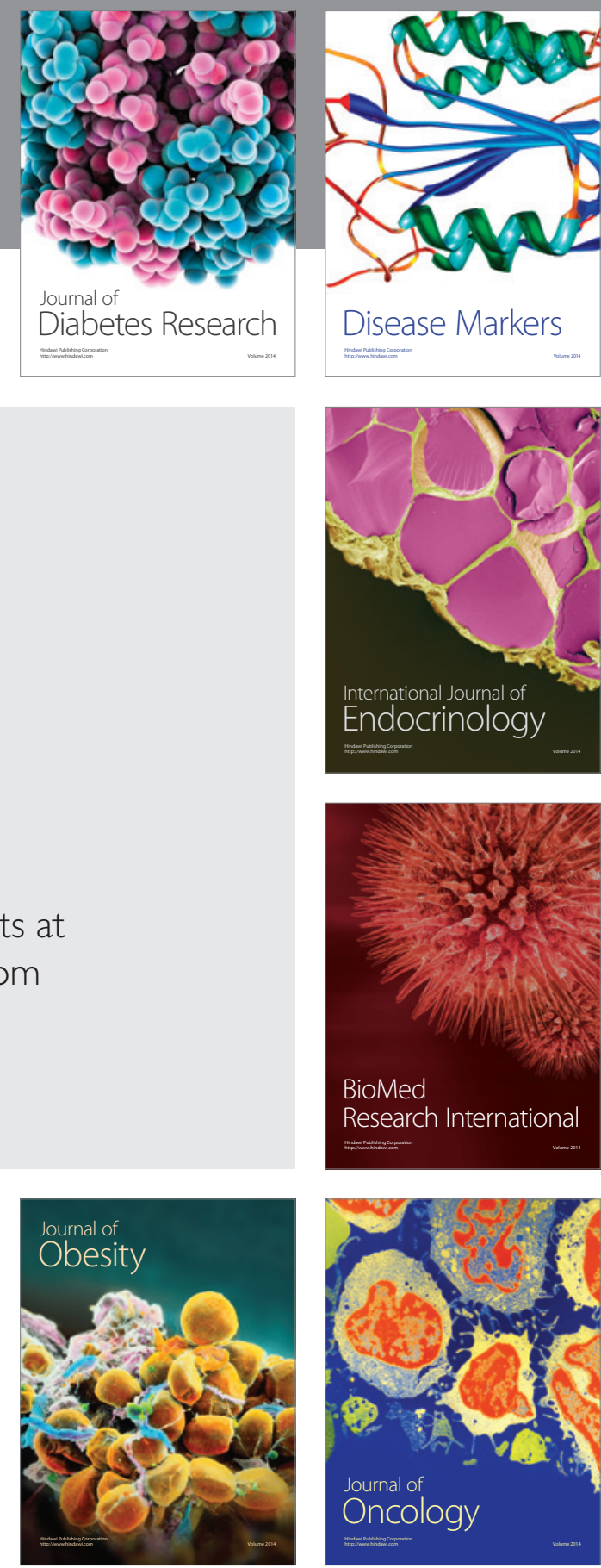

Disease Markers
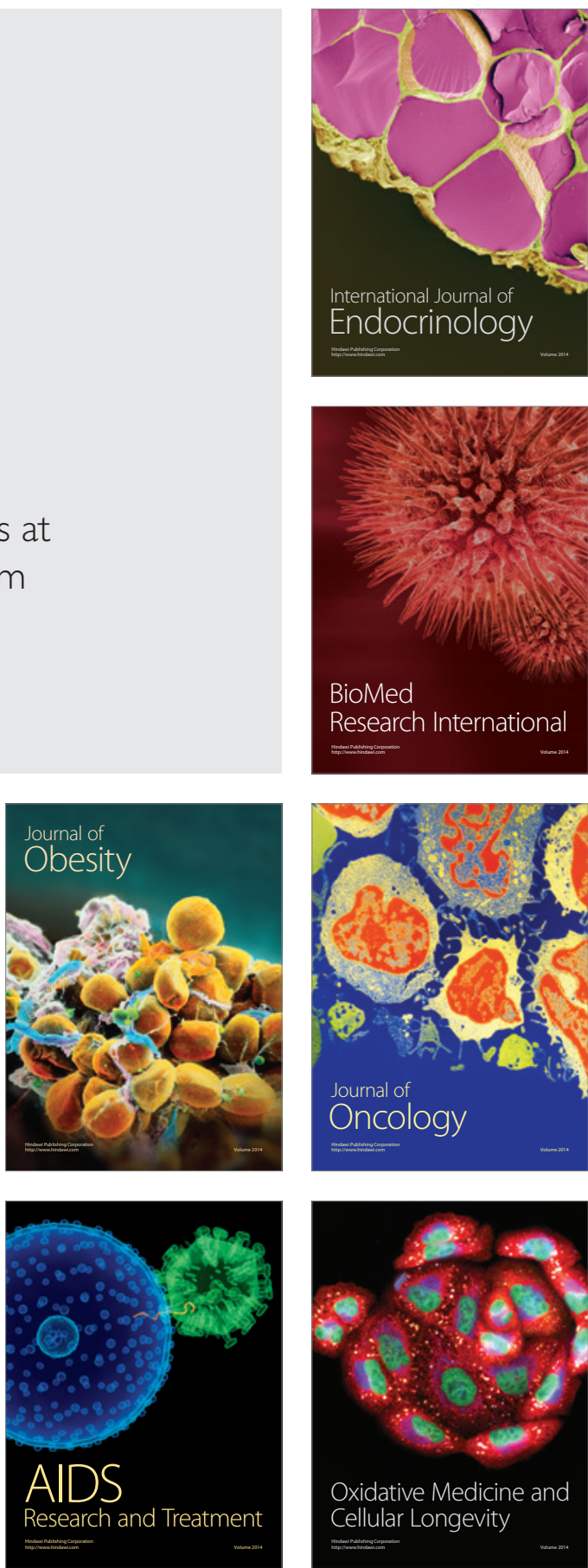